\title{
Late Holocene history of woodland dynamics and wood use in an ancient mining area of the Pyrenees (Ariège, France)
}

\author{
Vanessa Py-Saragaglia a, *, Raquel Cunill Artigas a, Jean-Paul Métailié a , Bruno Ancel ${ }^{\text {b, c }}$, \\ Sandrine Baron ${ }^{\mathrm{b}}$, Sandrine Paradis-Grenouillet ${ }^{\mathrm{d}, \mathrm{e}}$, Émilie Lerigoleur ${ }^{\mathrm{a}}$, \\ Nassima Badache ${ }^{a}$, Hugues Barcet ${ }^{a}$, Didier Galop ${ }^{a}$ \\ a GEODE, LABEX DRIIHM, UMR5602 CNRS Université Toulouse Jean Jaurès, Maison de la Recherche, 5 allées Antonio Machado, 31058 Toulouse Cedex 1, \\ France \\ b TRACES, UMR 5608 CNRS Université Toulouse Jean Jaurès, Maison de la Recherche, 5 allées Antonio Machado, 31058 Toulouse Cedex 1, France \\ ' Service Culturel Municipal de L'Argentière-La Bessée, Mairie de L'Argentière, 05120 L'Argentière-La Bessée, France \\ d GEOLAB, UMR 6042, Université de Limoges, Site de Limoges, $39 E$ rue Camille Guérin 87036 Limoges Cedex, France \\ e Dipartimento di Beni Culturali: Archeologia, Storia dell'Arte, del Cinema e della Musica, Padova University, Piazza Capitaniato 7, Padova, Italy
}

\section{A R T I C L E I N F O}

\section{Article history:}

Received 15 July 2016

Received in revised form

8 December 2016

Accepted 8 January 2017

Available online 1 March 2017

\section{Keywords:}

Mining

Charcoal kilns

Charcoal analysis

Woodland changes

Late Holocene

Eastern Pyrenees

\begin{abstract}
A B S T R A C T
This paper focuses on past woodland changes and land uses in an ancient mining area of the Eastern Pyrenees (Ariège, France). The area discussed is located at the western entrance of the Vicdessos, a valley with significant steel production, and it is crossed by the road used from the 14th c. to the end of the 18th c. for the iron-charcoal exchange with the forest Province of Couserans. The introduction of this singular exchange and the silver ore mining history of this border area raise the question of their impact on forest cover changes and changes in human practices and their link with anthropisation processes. To deal with this issue, we put in place an interdisciplinary approach involving archaeology, charcoal analysis, ecological history and geochemistry. The archaeological investigations and fourteen radiocarbon ages allowed characterising and dating of mining and charcoal-making remains. They situate the emergence of metal ore mining during the Second Iron Age and charcoal making activity between the 15th and 17th c. The geochemical analysis of 9 galena samples showed some different isotopic signatures between ores extracted in ancient times and those mined during the modern period. The charcoal analysis of (i) 2442 charcoals from 31 charcoal kilns (ii) 500 from one pedoarchaeological pit excavated in a waste heap related to firesetting, and (iii) 250 from two pedoanthracological sampling points carried out in the charcoal burning forest, permits a detailed reconstruction of the woodland cover changes from the Second Iron Age to the 19th c. Furthermore, the combination of data from different disciplines allows for a long-term reconstruction of human practices history and woodland management for different uses. In particular, the results show the transformation of the fir-beech forest, still dominated by fir around the turn of the Roman era, into pure beech wood managed on northern slopes for human daily needs, occasionally mining, lumber and mainly charcoal production until the 19th $\mathrm{c}$. The elimination of fir dates back to the 17th $\mathrm{c}$. This assumes the end of lumber activities in that period. Pedoanthracological and palynological data suggest that southern slopes, progressively deforested since the Bronze Age, were entirely devoted to permanent agropastoral activities probably at least since the end of the medieval period.
\end{abstract}

๑) 2017 Elsevier Ltd and INQUA. All rights reserved.

\section{Introduction}

In the past, mining and ore processing activities in Europe

\footnotetext{
* Corresponding author.

E-mail address: vanessa.py@univ-tlse2.fr (V. Py-Saragaglia).
}

exerted a strong pressure on woodland cover and contributed significantly to environmental changes (Monna et al., 2004a; Breitenlechner et al., 2010; Pèlachs et al., 2009; Ludemann, 2010; Knapp, 2012; Roepke and Krause, 2013; Py et al., 2014; Knapp et al., 2015; Tolksdorf et al., 2015). In the 80-90s, several studies carried out in the Eastern Pyrenees (southwestern France) and focused on charcoal making activity showed the importance of this 
phenomenon (Bonhôte and Vernet, 1988; Métailié et al., 1988; Bonhôte and Fruhauf, 1990; Izard, 1993; Davasse and Galop, 1994; Davasse, 2000; Bonhôte et al., 2002). However, with some exceptions (Dubois and Métailié, 2001), the data obtained for several valleys remained partial and disparate, particularly for periods prior to the 17th c. The most efficient way to understand the different anthropogenic factors affecting an ancient mining territory is to utilise a global and integrated approach as it was successfully done in the Basque Country (Galop et al., 2001; Monna et al., 2004b), Mont-Lozère (Servera Vives et al., 2014; Bal et al., 2015; Paradis-Grenouillet et al., 2015) and Harz Mountains (Knapp et al., 2013). Our present study is a continuation of the research conducted in the Eastern Pyrenees in the goal of deepening our knowledge of two areas with fragile mountainous ecosystems, which are particularly vulnerable to the effects of global change: the Upper Vicdessos and Couserans (Fig. 1). The first site contained rich iron ore deposits that were the subject of an important mining activity in the Rancié Massif from the Roman era (AD 3-5th c.) to the beginning of the last century (Dubois and Métailié, 2001; Dubois, 2001). The second area, located in the neighbouring Province of Couserans and called Aulus Mountain (Upper Garbet Valley), hosted an important silver mining district. In both of these areas, texts from the 14th c. suggest that mining and ore processing activities generated a significant consumption of wood, which has increased with the emergence of the mouline technology starting in the late 13th c. (Verna, 1994, 2001, 2008; Leroy et al., 2012). For historians, this kind of bloomery using hydraulic force seems to have been responsible for the first forest crisis, as is suggested by certain texts from the 14th c. (Verna, 2001). But, in our study area, it seems as though it worked to the contrary. In AD 1347-1348, an exchange treaty, sealing the fate of both these mining regions, consisted in an agreement to exchange iron from Vicdessos mines against charcoal from Couserans forests in order to safeguard wood resources of the former and to promote the development of the steel industry for the latter. But the real longterm implication of this treaty on Couserans' forest history and evolution is not well known and historical documentation prior to the 19th c. is sparse (Davasse, 2000). In addition, the paleoenvironmental studies that have been published on the subject are old and rare (Kenla, 1977; Kenla and Jalut, 1979; Reille, 1993). More recently, a palynological study of a peat core included in our study area (Galop, 1998; see Fig. 2 for the coring site location), but this sequence covers all the post-glacial period and its upper part is consequently compressed and does not provide sufficient temporal resolution to determine the accurate anthropisation processes such as mining, which were contemporary with historical periods. Consequently, this small area has focused our attention on the first part of our study, whose first results are reported here. Its history, closely tied to mining activities, raises the question of their impact on forest cover changes, human practices and anthropisation processes. In addition, we aim to characterise the implication of the iron-charcoal treaty on Couserans' forest history and evolution, in particular in our study area. In the context of the HumanEnvironment observatory Pyrenees-Vicdessos (Labex DRIIHM), we

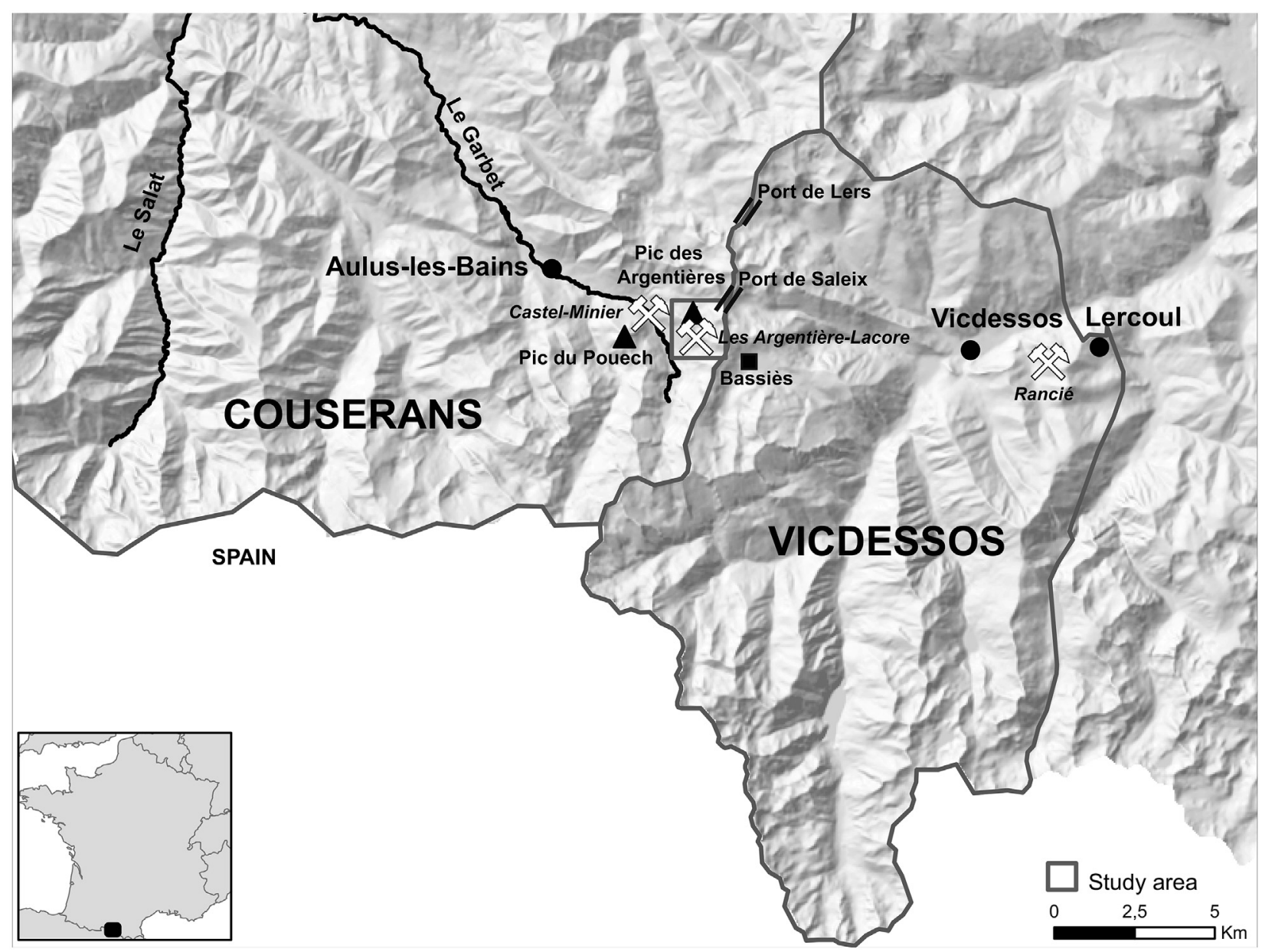

Fig. 1. National and regional location map of the study area discussed in this paper. 


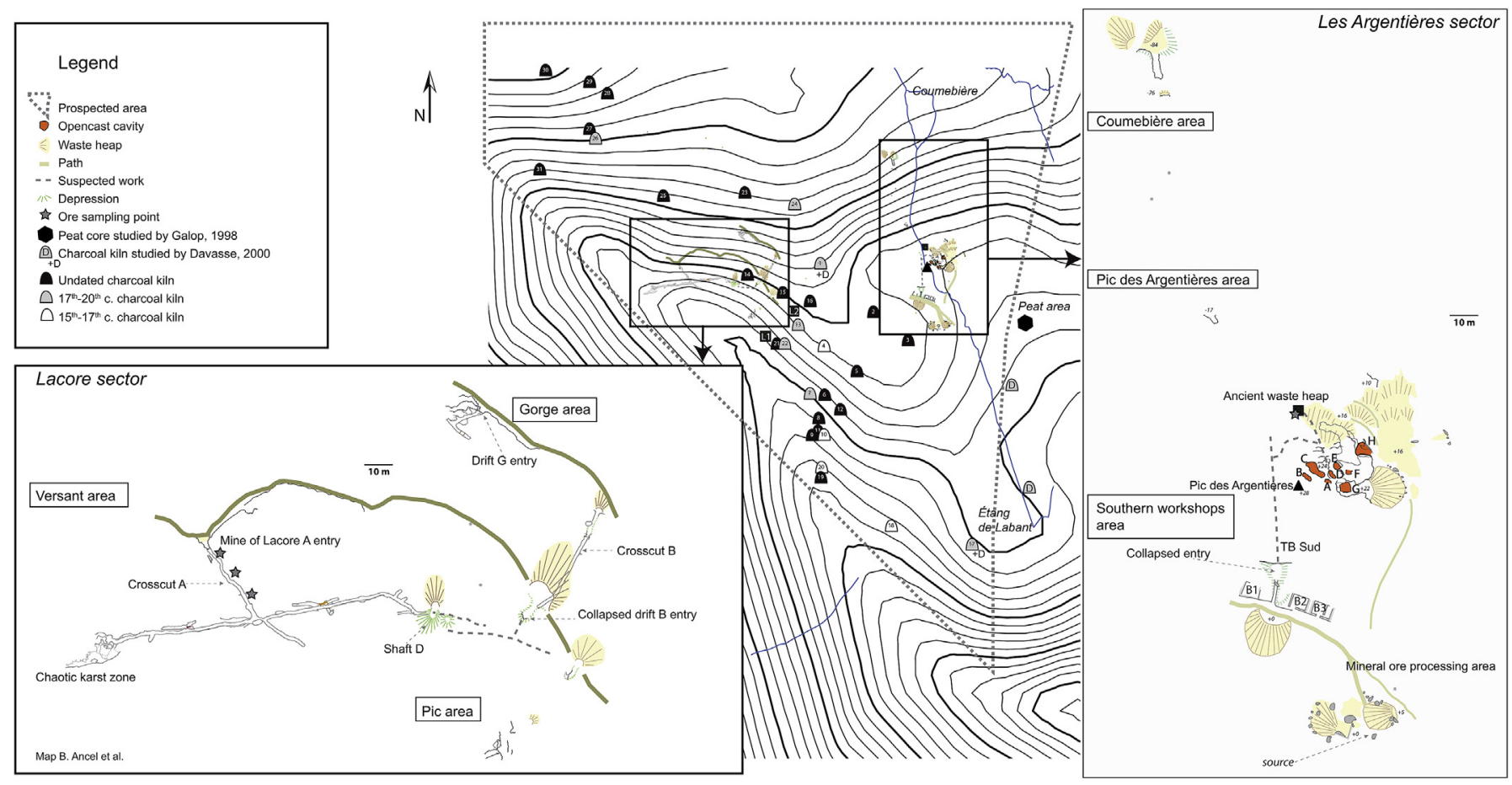

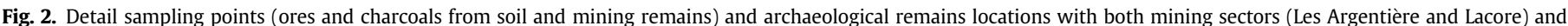
charcoal kilns.

put in place an interdisciplinary approach involving archaeology, charcoal analysis, ecological history and geochemistry, in order to answer these issues and (i) to characterise and date the archaeological remains of mining and charcoal-making; (ii) to determine the geochemical imprint of lead ores that were exploited locally; (iii) to identify forest resources exploited for mining and charcoalmaking; (iv) to understand their impact on soil charcoal concentration and anthracological assemblages. Lastly, we aim to offer new and additional insights into long term Pyrenean forest history in relation with human activities and practices.

\section{Regional setting}

\subsection{Geographical and geological contexts}

The Couserans province covers a group of convergent Pyrenean valleys opening onto the Salat catchment basin. This large mountain area is situated in the central part of the Pyrenees and shares $30 \mathrm{~km}$ of common border with Spain (Fig. 1). The Upper Garbet Valley forms its southeast extremity. This valley is backed by the Bassiès granitic massif and receives strong northwest winds and precipitation. Annual precipitation can reach $1.600 \mathrm{~mm}$ per year in the Upper Garbet Valley. On the high mountain slopes $2500 \mathrm{~mm}$ can be attained. This well-watered valley lodges the Aulus-lesBains town which is located at the end of two glacial valleys; the Upper Garbet Valley in the east and the Ars Valley in the west. These valleys are separated by the Pic du Pouech (1.737 $\mathrm{m}$ a.s.l.) (Fig. 1). The Upper Garbet valley marks the western border of a polymetallic area that presents most of the Pyrenees' ore resources. Beyond this metallogenic boundary, from the Vicdessos Valley to the Canigou Mountains, the iron ores are predominant. In the Aulus Mountain, polymetallic ore deposits are essentially hosted in Devonian dolomites. This area also hosted one of the most important silver mines called "Castel-Minier" (Fig. 1). These mineralisations were remobilised in fractures during the Hercynian folding and the simultaneous rise of a granite massif. The Pyrenean orogeny might have contributed to the geological and metallogenic complexity of this area. In total, about thirty mines were reported by the BRGM (French geological survey) in the Aulus Mountain (BRGM, 1983). In the geographical window of our study, on the right bank of the Garbet River, the main studied mines are named "Argentières" and "Laquorre" or "Lacore". The Argentières mining sector consists of two peaks that reach $1.600 \mathrm{~m}$ a.s.l. (Pic des Argentières) (Figs. 1 and 2). The main mineralisation consists of cerussite $\left(\mathrm{PbCO}_{3}\right)$, massive and dispersed galena $(\mathrm{PbS})$, grey copper (copper associated with galena presenting rich silver and antimony content), and numerous alteration minerals (BRGM, 1983). For a detailed and updated review of the ores implementation precisely in this study area, please refer to Munoz et al., 2016.

\subsection{Historical and archaeological contexts}

For both mining sectors, manuscripts attest several short periods of mining activity in the 18th (1773-1782) and 19th c. (1835-1842, 1857-1859, 1898-1899) (Dietrich, 1786; Mussy, 1864; Dubatik, 1981; Dubois, 1999; BRGM, 1983). Former mining phases were suspected by Dubois (1999) but never attested by archaeological evidence or geochemical imprints (Dubois and Métailié, 1991). Davasse (2000) studied in this area four kiln sites of which two are reviewed here (CK 1, 17) (Fig. 2). In the Lacore sector, mining works were more extensive, particularly underground. Geologists and archaeologists have always connected them to the modern period (Dietrich, 1786; Mussy, 1864; Passaqui, 1964; Dubatik, 1981; BRGM, 1983). This chronology is disconnected from the Castel-Minier site, located farther down in the valley (Fig. 1), where the main mining phases took place between the 13th c. and the end of the 14th c. The mouline exploited in the same area was operated from the 14th to the 16th c. (Dillmann et al., 2006; 
Téreygeol, 2007; Téreygeol et al., 2010).

The iron-charcoal treaty signed by the Count of Foix, the viscount of Couserans and the community of Vicdessos, is known through three corrupt copies made in the 18th c. and the original document is almost illegible (Verna, 2001). In these copies, the treaty appears to have been reached in favour of Vicdessos. In exchange for supplying iron for Viscount Couserans' moulines, the Vicdessos people (especially the master ironworkers) obtained the right to make charcoal in Couserans forest without any limitation. Only the cutting of oaks and young trees measuring "one hand" was prohibited. Verna (2001) assumes the existence of other conditions intentionally left out in the later copies. The mule drivers with loads (charcoal, timber wood and iron ore) used the mountain pass of Port de Saleix (Fig. 1) located just beside Les Argentières-Lacore mines and that of Port de Lers further north. In the same place, on the Coumebière Plateau, there are also a lot of remains of agropastoral structures, such as huts and enclosures, which are evidence of centuries-old pastoral activities.

\subsection{Current woodland}

Currently, the Argentières sector located on the southern side occupies an area quasi-deprived of trees. Beech wood borders expand on the margins of the Plateau de Coumebière and the southern lateral moraine of the Étang de Labant. The dominant vegetation consists of wet grassland: dwarf ericaceous shrubs (Rhododendron ferrugineum L., Vaccinium myrtillus L., Calluna vulgaris L. Hull), juniper heath patches (Juniperus communis subsp. nana (Hook.) Sym), and a few willows and mountain ashes (Sorbus aucuparia $\mathrm{L}$.). The northern side vegetation is dominated by beech wood (Fagus sylvatica L.) with high-growing coppices of old trees and isolated fir (Abies alba Mill.) trees in regeneration. More dense fir patches mixed with beeches constitute the treeline at 1.650-1.700 m. a.s.l. High altitudes pioneer species (Pinus uncinata Ramond ex DC., Betula) are almost absent from ancient pastoral areas. By contrast, the beech regeneration is very strong on the edges of intermediate pastures (Plateau de Coumebière).

\section{Material and methods}

\subsection{Field survey}

Detailed documentary research was conducted in historical archives and literature before the field investigation was carried out in 2013-2016 (Py et al., 2013a). Archaeological prospecting has focused within the area bound by the Plateau de Coumebière in the north (and including it), Lacore Ridge in the west, Labant Cirque in the south and Argentières peaty area in the east (Fig. 2). All mining remains identified were the subject of observations, descriptions, surface and underground topographical surveys (Py et al., 2013a). The main surface topographic points were geo-referenced with a differential GPS. Prospecting has focused on the Lacore side, and also around the Pic des Argentières and near the trail that leads to the Étang de Labant (a total area of 50 ha) because these areas provide mining remains (Fig. 2). This prospected zone was locked down from the foot of the sides up to the top of the ridges. All topographic (flat terraces, small depressions) and phytological indices (vegetation changes) are tested with a pedological hand auger. The demonstrated kiln sites were recorded, described, drawn to scale, photographed and geo-referenced. The data recorded were integrated in a Geographic Information System (GIS). In the short term we wanted to enlarge the investigated area at the east of the Pic des Argentières.

\subsection{Sampling protocol}

\subsubsection{Charcoal Kilns (CK)}

A study of a single or few $\mathrm{CK}$, even if it is exhaustive, provides incomplete information. In order to obtain a broad spatial vision, we have chosen to sample all CK using pedological core sampling. The results obtained in this present study show that two sampling points are sufficient to determine the anthracological composition of a CK. But three and four supplementary points may be required to obtain a sufficient amount of charcoal for the initial diameter estimation of the wood. These points are located between the central part and lower border of the terraces in a way that avoids the sharp break with the upstream side that is marked by colluvial deposits. The sampling was performed in intervals of $20 \mathrm{~cm}$ in depth, which is governed by the hand auger length. The coring was stopped when the degraded substratum level (ochre) had been reached. In order to avoid contamination, the hole and the auger were cleaned between each sample. All samples were sieved with water in a column of sieves with 4 and $2 \mathrm{~mm}$ meshes.

\subsubsection{Mining works}

3.2.2.1. Charcoal sampling. The detailed examination of the mining remains on the Pic des Argentières resulted in the identification of innovative firesetting marks i.e. rounded working face fragments (Fig. 2, Les Argentière sector, $\mathrm{C}$ ) and charcoal particles within the waste heaps spread out on the hill side $10 \mathrm{~m}$ down. In order to date and characterise this mining phase prior to the use of explosive powder, a pedoarchaeological pit ( $0.6 \mathrm{~m}$ wide and $1.30 \mathrm{~m}$ deep) was opened in the top part of the waste heaps (Fig. 3). The excavation was stopped when the altered limestone substratum were found. A cross-section has revealed 6 Stratigraphic Units (SU) described in the Fig. 3. Five litres of materials have been taken in the SU $0,1,3$ and 4 for charcoal analysis. The SU 2 being only decimetre blocks was not sampled. All samples were sieved with water in a column of sieves with 5,2 and $0.8 \mathrm{~mm}$ meshes.

3.2.2.2. Ores sampling. To characterise the geochemical imprints of the different mining phases and to create a lead isotopic database of local metallic resources exploited during ancient times, several ore pieces were sampled. One set of ores is from the ancient waste heaps located at Les Argentières (on the SU 4 surface) (Figs. 2 and 3), and one from the Lacore crosscut residual vein (Fig. 2). The lead isotopic analyses have been conducted on these samples to understand the implementation of ores in this wide mining district situated in the Pyrenean Mountains. The protocol and method are reported in Munoz et al., 2016.

\subsubsection{Pedoanthracological pits}

Two pedoanthracological pits were opened in the Lacore slope (northeast-facing), with the goal of characterising the potential impact of charcoal burning activities on the charcoal assemblages within soils (Fig. 2). A first sampling point (Lacore 1) is located a few metres below the Lacore ridge within a beech wood at $1.578 \mathrm{~m}$ a.s.l., and next to the CK 21 and 22 (Fig. 2). Locally, the bedrock is limestone. The slope is quite steep (70-80\%) and granitic rock outcrops are located near it. The pit has been dug $100 \mathrm{~cm}$ deep. It showed a simplified pedological structure with two main soil horizons A and B (1-2) (Fig. 4). Four levels have been sampled for pedoanthracological analysis: I $(0-6 \mathrm{~cm})$, II $(6-20 \mathrm{~cm})$, III $(20-70 \mathrm{~cm})$ and IV $(70-100 \mathrm{~cm})$. The second sampling point (Lacore 2 ) is located at the foot of the same slope, $30 \mathrm{~m}$ below and next to the CK 13 (Fig. 2). The total depth of the pit is $90 \mathrm{~cm}$. The pedological structure is similar to the first one with two horizons A and B (1-2) (Fig. 4). Four levels have been sampled: I $(0-17 \mathrm{~cm})$, II $(17-45 \mathrm{~cm})$, III 
Stratigraphic section of the ancient waste heap Les Argentières sector

SUO: dump surface leached with charcoals.

SU1: earthy matrix with fine gravels, quartz inclusions and charcoals.

2: limestone blocks with earthy inclusions from SU1.

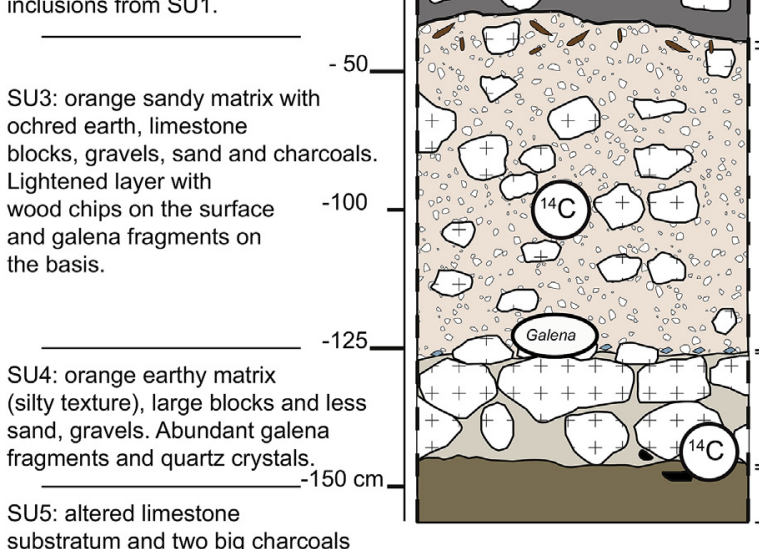
substratum and two big charcoals at the interface with SU4.

\section{Anthracological spectra}
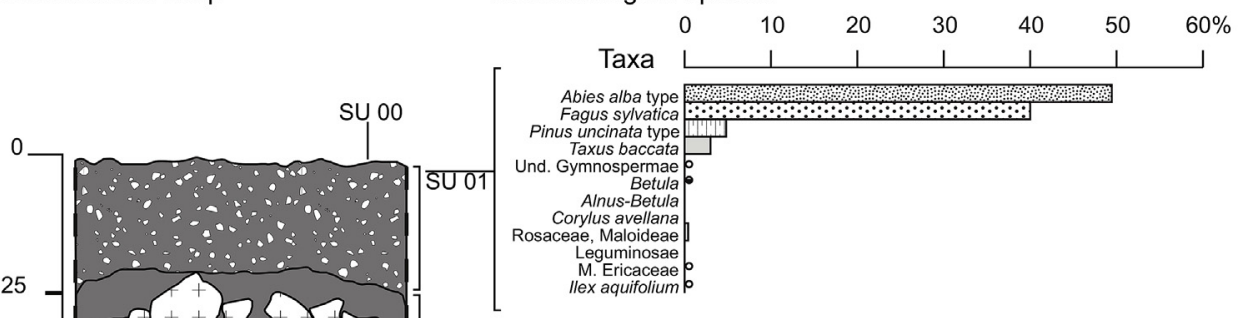

SU 02

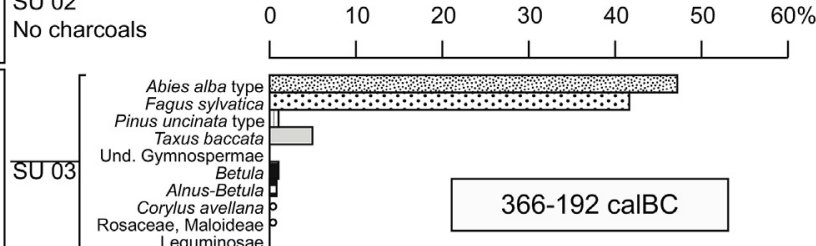

Rosaceae, Maloideae

Leguminosae

Ilex aquifolium

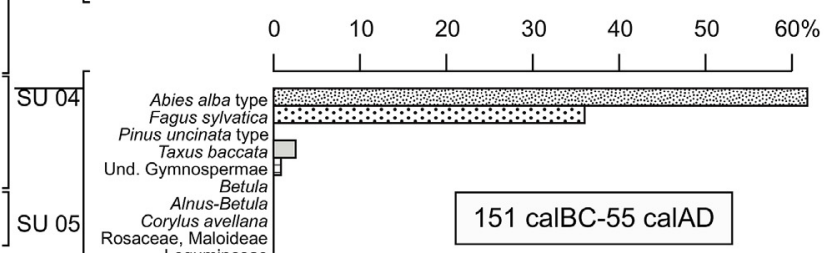

$\mathrm{n}=500$

$\circ \leq 0.5 \%$

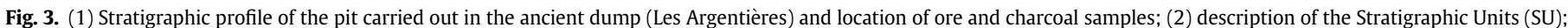
(3) percentage anthracological spectra.

$(45-74 \mathrm{~cm})$ and IV $(74-90 \mathrm{~cm})$. Isolation of soil charcoals was based on a procedure previously published by Talon et al. (1998).

\subsection{Laboratory analyses}

\subsubsection{Charcoal analysis}

3.3.1.1. Charcoals from charcoal kilns (CK). Fragments from the $4 \mathrm{~mm}$ mesh were analysed. The $2 \mathrm{~mm}$ fraction was kept for a future study focusing on differential fragmentation of main taxa (Fagus sylvatica and Abies alba). A minimum of 50 fragments per core was analysed (exceptionally less for the second sampling level in CK 30). A smaller number of fragments per core was analysed when several sampling points were sampled within the same site (Table 1). The transversal cut was performed in such a way that it could be as complete as possible. The radial and tangential cuts were carried out into the other part or breakage wastes. The microscopic observation and taxonomic identification were carried out using a reflected light microscope equipped with a 100, 200, 500 and 1000 magnification lens (Leika DM4), and with the help of xylology atlases (Schweingruber, 1978, 1990; Vernet et al., 2001) and also charred wood references of the GEODE laboratory.

Statistical analyses were performed using $\mathrm{R}$ software version 3.3.1 (R Development Core Team, 2015) with the "Rcmdr" package (Fox et al., 2016), the FactoMineR plugin (Husson et al., 2016) and the "ggplot2" package (Wickham et al., 2016). First, a Hierarchical Clustering on Principal Components (HCPC) was carried out in order to gather the CK into a couple of clusters which would correspond to different consumption profiles (i.e. different group levels of fir/beech proportions per core). Then we calculated the pairwise comparisons between these independent clusters respectively for fir and beech, using Wilcoxon rank sum test (ie. pairwise.wilcox.test $\mathrm{R}$ function). This nonparametric test (also called MannWhitney test) is applied on small sample size with the null hypothesis that two samples come from the same population.

When the bark was preserved, the growth arrest zone was identified (early wood, end of early wood, late wood and end of late wood) as a way to determine cutting seasons (Py-Saragaglia et al., 2015). The minimal initial diameter of charred wood was measured with a sliding caliper when pith and bark were preserved. But in most cases, this measure was not possible. In order to establish an estimate of the initial diameter, we measured the radius curvature (distance between the last visible ring and the pith, i.e. the heart wood) on charcoal using a macroscope (Leika ZAPO) connected to a computer running the software "AnthracoLoJ" developed by the GEOLAB laboratory (Paradis-Grenouillet et al., 2010; 2015). This dendroanthracological approach is still being perfected.

On the basis of previous studies interested in characterising the quality and state of the wood before carbonisation (Théry-Parisot, 2001; Marguerie and Hunot, 2007; Moskal-del Hoyo et al., 2010; Théry-Parisot and Henry, 2012; Py et al., 2013b; Henry and ThéryParisot, 2014), we carried out other examinations. The presence of reaction wood has been recorded for conifers. Parasite traces mainly fungal hyphae - and anatomical deformations related to fungal decay infestation (cells collapsed) have been sought within different anatomical planes. Vitrified charcoals (partially or totally) and those with radial cracks were sought. On the basis of the protocol described in Py et al. (2013b), 12 samples were selected for radiocarbon dating (Table 2 ).

3.3.1.2. Charcoals from soils. Anthracomass was calculated on the basis of the mass of charcoal fragments larger than $0.8 \mathrm{~mm}$ (Table 3) (Cunill et al., 2015). A taxonomic analysis was done for all charcoal extracted $(n=108)$ in Lacore 1 due to the low quantity of charcoal present in this sampling point. In the case of Lacore 2, the totality of charcoals from sampling levels 2,3 , and 4 were analysed for the 

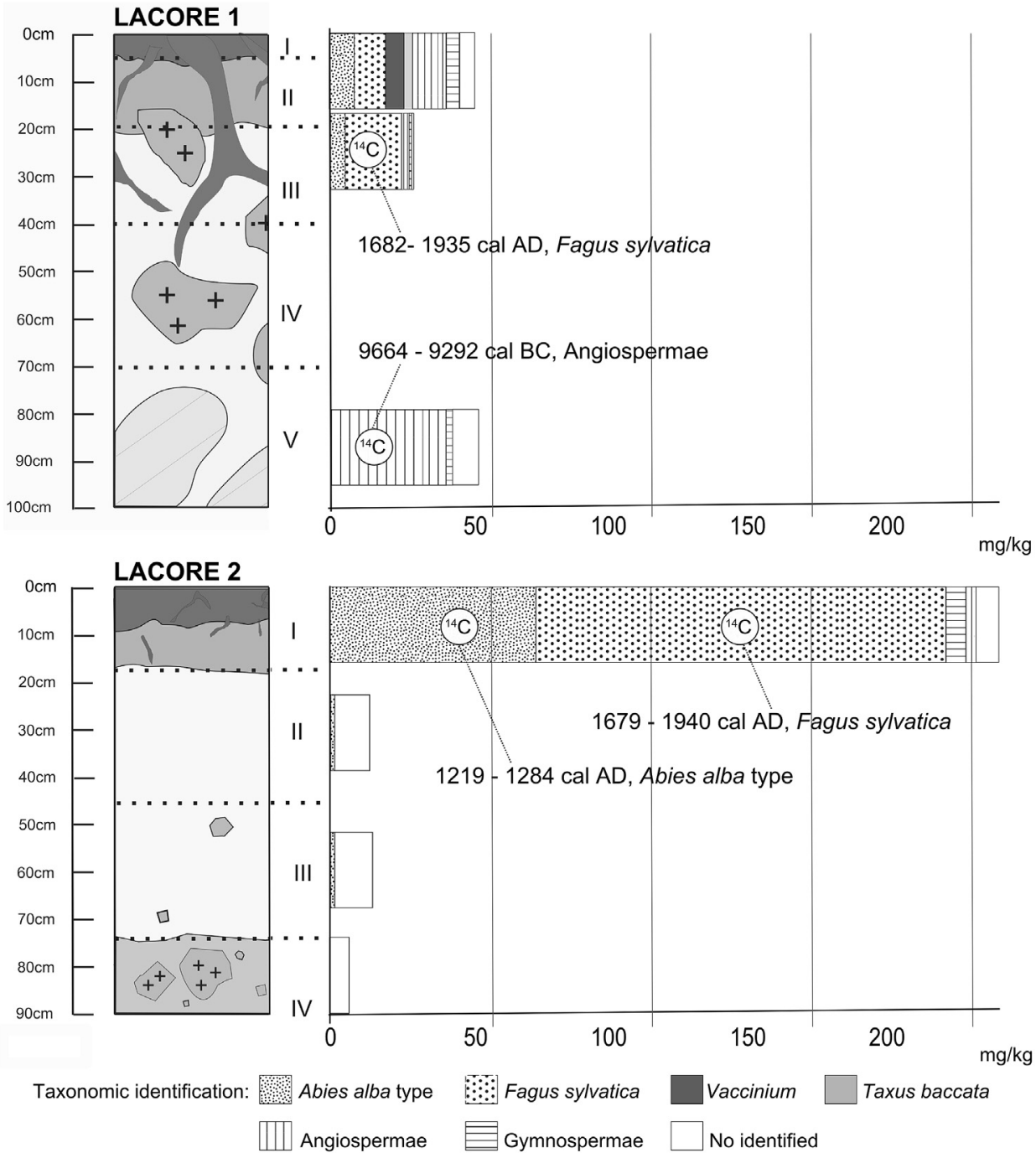

Fig. 4. Soil profiles and specific anthracomass per profile (SAP)* and per identified taxa (SAT) (in milligrams per kilogram) of Lacore 1 and Lacore $2 .{ }^{*}$ Please refer to Touflan et al., 2010.

same reason $(\mathrm{n}=15) .100$ pieces from $0.8 \mathrm{~mm}$ mesh were randomly selected and a total of 127 fragments were analysed from the sampling level 1. A total of 312 charcoal fragments were isolated from Lacore 2. In order to avoid bias due to the potential differential fragmentation of main taxa, charcoals from each mesh ( $>5 \mathrm{~mm}, 5-$ $2 \mathrm{~mm}$, and $2-0.8 \mathrm{~mm}$ ) were analysed separately. Fir and beech charcoals infested by hyphae fungi and/or vitrified were counted (Table 3). A total of 4 samples were selected for radiocarbon dating (Table 2 and Fig. 4).

3.3.1.3. Charcoals from mine waste heaps. Their study protocol is based on previous works (Py, 2006; Py et al., 2013b, 2014). All fragments from the $5 \mathrm{~mm}$ mesh and the majority of those from $2 \mathrm{~mm}$ have been analysed in order to identify a minimum of 100 fragments per SU $(n=500)$. The $0.8 \mathrm{~mm}$ non-passing fraction is sorted to retrieve charcoals, seeds, microfauna and insect remains. It is currently being studied. The Pareto index has been calculated for each SU and also the entire corpus (Table 4) (Chabal, 1997). These charcoals being small, they were not investigated in terms of dendroanthracology but other observations were feasible (presence of hyphae, vitrifications and radial cracks) (Table 4). Two samples from the base of SU 4 and the main body of SU 3 were selected for radiocarbon dating (Fig. 3 and Table 2).

\subsubsection{Radiocarbon dating}

Charcoal samples selected for radiocarbon dating (total of 18) have been dated by Accelerator Mass Spectrometry (AMS) in the Center for Applied Isotope Studies (UGA, US) and Poznan Radiocarbon Laboratory (Poznan, Poland) (Table 2). The radiocarbon ages were calibrated using the Oxcal program, version 4.2 based on the Intcal 13.14c database (Reimer et al., 2013) and with a standard deviation of 2 sigma (95\% probability).

\section{Results}

\subsection{Archaeological evidences}

\subsubsection{Ancient mining sectors and related $\mathrm{Pb}$ isotopic signatures of} ores

Les Argentieres sector. The crossing of historical sources and archaeological data suggests that mining remains identified in the field mainly correspond to the 18th c. The activity in the 19th c. has consisted principally in clearing and scraping former works. The main mining remains were distributed in three areas (Fig. 2): the 
Table 1

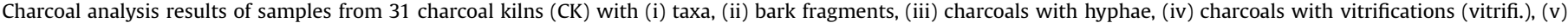
conifers with compression wood (CW), (vi) charcoals with radial cracks (RC) absolute (n) and relative frequencies (\%).

\begin{tabular}{|c|c|c|c|c|c|c|c|c|c|c|c|c|c|c|}
\hline CK [depth-S] & $\begin{array}{l}\text { Abies } \\
\text { alba t. }\end{array}$ & $\begin{array}{l}\text { Fagus } \\
\text { sylvatica }\end{array}$ & Fraxinus & M. Ericaceae & $\begin{array}{l}\text { Taxus } \\
\text { baccata }\end{array}$ & U. Angio. & U. Gymno. & Total & bark & Total & w/hyphae & w/vitrifi. & $\begin{array}{l}\text { Gymno. } \\
\text { w/CW }\end{array}$ & $\mathrm{w} / \mathrm{RC}$ \\
\hline $1[0-20 \mathrm{~cm})$ & 3 & 155 & 0 & 0 & 1 & 0 & 0 & 159 & 16 & 175 & 86 & 45 & 1 & 13 \\
\hline$(\%-\mathrm{H})$ & 1.9 & 97.5 & 0 & 0 & 0.6 & 0 & 0 & - & 9.1 & - & 54.1 & 28.3 & 25 & 7.4 \\
\hline $2[0-20 \mathrm{~cm})$ & 1 & 92 & 0 & 0 & 0 & 0 & 0 & 93 & 7 & 100 & 81 & 24 & 0 & 1 \\
\hline$(\%-\mathrm{H})$ & 1.1 & 98.9 & 0 & 0 & 0 & 0 & 0 & - & 7 & _- & 87.1 & 25.8 & 0 & 1 \\
\hline $3[0-20 \mathrm{~m}]$ & 24 & 59 & 0 & 1 & 0 & 0 & 1 & 85 & 15 & 100 & 56 & 20 & 4 & 4 \\
\hline$(\%-\mathrm{H})$ & 28.2 & 69.4 & 0 & 1.2 & 0 & 0 & 1.2 & - & 15 & - & 65.9 & 23.5 & 16 & 4 \\
\hline $4[0-20 \mathrm{~cm}]$ & 5 & 68 & 0 & 0 & 0 & 0 & 0 & 73 & 2 & 75 & 52 & 14 & - & - \\
\hline$(\%-H)$ & 6.8 & 93.2 & 0 & 0 & 0 & 0 & 0 & - & 2.7 & - & 71.2 & 19.2 & - & - \\
\hline $4[20-40 \mathrm{~cm}]$ & 29 & 51 & 0 & 0 & 0 & 0 & 0 & 80 & 2 & 82 & 42 & 10 & - & - \\
\hline$(\%-\mathrm{H})$ & 36 & 64 & 0 & 0 & 0 & 0 & 0 & - & 2 & - & 53 & 13 & - & - \\
\hline $\mathrm{T}-4$ & 34 & 119 & 0 & 0 & 0 & 0 & 0 & 153 & 4 & 157 & 94 & 24 & 8 & 1 \\
\hline$(\%-\mathrm{H})$ & 22.2 & 77.8 & 0 & 0 & 0 & 0 & 0 & _ & 3 & _- & 61 & 16 & 23.5 & 0.6 \\
\hline $5[0-20 \mathrm{~cm}]$ & 3 & 41 & 0 & 0 & 0 & 0 & 1 & 45 & 5 & 50 & 23 & 17 & 1 & 2 \\
\hline$(\%-\mathrm{H})$ & 6.7 & 91.1 & 0 & 0 & 0 & 0 & 2.2 & - & 10 & - & 51.1 & 37.8 & 25 & 4 \\
\hline $6[0-20 \mathrm{~cm}]$ & 5 & 39 & 0 & 0 & 0 & 0 & 1 & 45 & 5 & 50 & 27 & 27 & 0 & 2 \\
\hline$(\%-\mathrm{H})$ & 11.1 & 86.7 & 0 & 0 & 0 & 0 & 2.2 & - & 10 & - & 60 & 60 & 0 & 4 \\
\hline $7[0-20 \mathrm{~cm}]$ & 2 & 43 & 0 & 0 & 0 & 1 & 0 & 46 & 4 & 50 & 16 & 23 & - & - \\
\hline$(\%-\mathrm{H})$ & 4.3 & 93.5 & 0 & 0 & 0 & 2.2 & 0 & - & 8 & - & 34.8 & 50 & - & - \\
\hline $7[20-40 \mathrm{~cm}]$ & 6 & 43 & 0 & 0 & 0 & 0 & 0 & 49 & 1 & 50 & 26 & 15 & - & - \\
\hline$(\%-\mathrm{H})$ & 12.2 & 87.8 & 0 & 0 & 0 & 0 & 0 & - & 2 & - & 53 & 30.6 & - & - \\
\hline $\mathrm{T}-7$ & 8 & 86 & 0 & 0 & 0 & 1 & 0 & 95 & 5 & 100 & 42 & 38 & 2 & 4 \\
\hline$(\%-\mathrm{H})$ & 8.4 & 90.5 & 0 & 0 & 0 & 1.1 & 0 & - & 5 & - & 44 & 40 & 25 & 4 \\
\hline $8[0-20 \mathrm{~cm}]$ & 5 & 44 & 0 & 0 & 0 & 0 & 0 & 49 & 1 & 50 & 45 & 13 & 0 & 8 \\
\hline$(\%-\mathrm{H})$ & 10.2 & 89.8 & 0 & 0 & 0 & 0 & 0 & - & 2 & - & 91.8 & 26.5 & 0 & 16 \\
\hline $9[0-20 \mathrm{~cm}]$ & 0 & 42 & 0 & 0 & 0 & 0 & 0 & 42 & 8 & 50 & 33 & 23 & - & 0 \\
\hline$(\%-\mathrm{H})$ & 0 & 100 & 0 & 0 & 0 & 0 & 0 & - & 16 & - & 78.6 & 54.8 & - & 0 \\
\hline $10[0-20 \mathrm{~cm}]$ & 18 & 31 & 1 & 0 & 0 & 0 & 0 & 50 & 0 & 50 & 40 & 17 & 1 & 1 \\
\hline$(\%-\mathrm{H})$ & 36 & 62 & 2 & 0 & 0 & 0 & 0 & - & 0 & - & 80 & 34 & 5.6 & 2 \\
\hline $11[0-20 \mathrm{~cm}]$ & 11 & 38 & 0 & 0 & 0 & 0 & 0 & 49 & 1 & 50 & 34 & 20 & 3 & 2 \\
\hline$(\%-\mathrm{H})$ & 22.4 & 77.6 & 0 & 0 & 0 & 0 & 0 & - & 2 & - & 69.4 & 40.8 & 27.3 & 4 \\
\hline $12[0-20 \mathrm{~cm}]$ & 0 & 46 & 0 & 0 & 0 & 0 & 0 & 46 & 4 & 50 & 32 & 26 & - & 3 \\
\hline$(\%-\mathrm{H})$ & 0 & 100 & 0 & 0 & 0 & 0 & 0 & - & 8 & - & 69.6 & 56.5 & - & 6 \\
\hline $13[0-20 \mathrm{~cm}]$ & 0 & 50 & 0 & 0 & 0 & 0 & 0 & 50 & 0 & 50 & 40 & 16 & - & - \\
\hline$(\%-\mathrm{H})$ & 0 & 100 & 0 & 0 & 0 & 0 & 0 & - & 0 & - & 80 & 32 & - & - \\
\hline $13[20-40 \mathrm{~cm}]$ & 0 & 46 & 0 & 0 & 0 & 0 & 0 & 46 & 4 & 50 & 18 & 23 & - & - \\
\hline$(\%-\mathrm{H})$ & 0 & 100 & 0 & 0 & 0 & 0 & 0 & - & 8 & - & 39.1 & 50 & - & - \\
\hline $\mathrm{T}-13$ & 0 & 96 & 0 & 0 & 0 & 0 & 0 & 96 & 4 & 100 & 58 & 39 & - & 1 \\
\hline$(\%-\mathrm{H})$ & 0 & 100 & 0 & 0 & 0 & 0 & 0 & - & 4 & - & 60.4 & 40.6 & - & 1 \\
\hline $14[0-20 \mathrm{~cm}]$ & 0 & 48 & 0 & 0 & 0 & 0 & 0 & 48 & 2 & 50 & 9 & 20 & - & - \\
\hline$(\%-\mathrm{H})$ & 0 & 100 & 0 & 0 & 0 & 0 & 0 & - & 4 & - & 18.75 & 41.7 & - & - \\
\hline $14[20-40 \mathrm{~cm}]$ & 3 & 47 & 0 & 0 & 0 & 0 & 0 & 50 & 0 & 50 & 29 & 33 & - & - \\
\hline$(\%-\mathrm{H})$ & 6 & 94 & 0 & 0 & 0 & 0 & 0 & - & 0 & - & 58 & 66 & - & - \\
\hline $\mathrm{T}-14$ & 3 & 95 & 0 & 0 & 0 & 0 & 0 & 98 & 2 & 100 & 38 & 53 & 0 & 4 \\
\hline$(\%-\mathrm{H})$ & 3.1 & 96.9 & 0 & 0 & 0 & 0 & 0 & - & 2 & - & 39 & 54 & 0 & 4 \\
\hline $15[0-20 \mathrm{~cm}]$ & 10 & 38 & 0 & 0 & 0 & 0 & 0 & 48 & 2 & 50 & 10 & 10 & 0 & 3 \\
\hline$(\%-\mathrm{H})$ & 20.8 & 79.2 & 0 & 0 & 0 & 0 & 0 & - & 4 & - & 20.8 & 20.8 & 0 & 6 \\
\hline $16[0-20 \mathrm{~cm}]$ & 9 & 81 & 0 & 0 & 0 & 0 & 0 & 90 & 10 & 100 & 66 & 34 & 1 & 10 \\
\hline$(\%-\mathrm{H})$ & 10 & 90 & 0 & 0 & 0 & 0 & 0 & - & 10 & - & 73.3 & 37.8 & 11.1 & 10 \\
\hline $17[0-20 \mathrm{~cm}]$ & 7 & 105 & 0 & 0 & 0 & 0 & 0 & 112 & 16 & 128 & 48 & 40 & - & - \\
\hline$(\%-\mathrm{H})$ & 6.3 & 93.8 & 0 & 0 & 0 & 0 & 0 & - & 12.5 & - & 42.9 & 35.7 & - & - \\
\hline $17[20-40 \mathrm{~cm}]$ & 5 & 38 & 0 & 0 & 0 & 0 & 0 & 43 & 7 & 50 & 10 & 25 & - & - \\
\hline$(\%-\mathrm{H})$ & 11.6 & 88.4 & 0 & 0 & 0 & 0 & 0 & - & 14 & - & 23.3 & 58.1 & - & - \\
\hline $\mathrm{T}-17$ & 12 & 143 & 0 & 0 & 0 & 0 & 0 & 155 & 23 & 178 & 58 & 65 & 0 & 14 \\
\hline$(\%-\mathrm{H})$ & 8 & 92 & 0 & 0 & 0 & 0 & 0 & - & 12.9 & - & 37 & 42 & 0 & 7.9 \\
\hline $18[0-20 \mathrm{~cm}]$ & 23 & 126 & 0 & 0 & 0 & 0 & 0 & 149 & 13 & 162 & 103 & 77 & 6 & 5 \\
\hline$(\%-\mathrm{H})$ & 15.4 & 84.6 & 0 & 0 & 0 & 0 & 0 & _- & 8 & - & 69.1 & 51.7 & 26.1 & 3.1 \\
\hline $19[0-20 \mathrm{~cm}]$ & 6 & 42 & 0 & 0 & 0 & 0 & 0 & 48 & 2 & 50 & 39 & 16 & 1 & 2 \\
\hline$(\%-\mathrm{H})$ & 12.5 & 87.5 & 0 & 0 & 0 & 0 & 0 & - & 4 & - & 81.3 & 33.3 & 16.7 & 4 \\
\hline $20[0-20 \mathrm{~cm}]$ & 8 & 37 & 0 & 0 & 0 & 0 & 1 & 46 & 4 & 50 & 35 & 10 & 0 & 6 \\
\hline$(\%-\mathrm{H})$ & 17.4 & 80.4 & 0 & 0 & 0 & 0 & 2.2 & _ & 8 & _ & 76.1 & 21.7 & 0 & 12 \\
\hline $21[0-20 \mathrm{~cm}]$ & 6 & 43 & 0 & 0 & 0 & 0 & 0 & 49 & 1 & 50 & 37 & 11 & 2 & 2 \\
\hline$(\%-\mathrm{H})$ & 12.2 & 87.8 & 0 & 0 & 0 & 0 & 0 & - & 2 & - & 75.5 & 22.4 & 33.3 & 4 \\
\hline $22[0-20 \mathrm{~cm}]$ & 0 & 49 & 0 & 1 & 0 & 0 & 0 & 50 & 0 & 50 & 40 & 15 & - & 3 \\
\hline$(\%-\mathrm{H})$ & 0 & 98 & 0 & 2 & 0 & 0 & 0 & - & 0 & - & 80 & 30 & - & 6 \\
\hline $23[0-20 \mathrm{~cm}]$ & 3 & 89 & 0 & 0 & 0 & 0 & 0 & 92 & 8 & 100 & 50 & 46 & 1 & 0 \\
\hline$(\%-\mathrm{H})$ & 3.3 & 96.7 & 0 & 0 & 0 & 0 & 0 & - & 8 & - & 54.3 & 50 & 33.3 & 0 \\
\hline $24[0-20 \mathrm{~cm}]$ & 0 & 45 & 0 & 0 & 0 & 0 & 0 & 45 & 5 & 50 & 28 & 19 & - & 1 \\
\hline$(\%-H)$ & 0 & 100 & 0 & 0 & 0 & 0 & 0 & - & 10 & - & 62.2 & 42.2 & - & 2 \\
\hline $25[0-20 \mathrm{~cm}]$ & 9 & 64 & 0 & 0 & 0 & 0 & 0 & 73 & 2 & 75 & 31 & 24 & 6 & 4 \\
\hline$(\%-\mathrm{H})$ & 12.3 & 87.7 & 0 & 0 & 0 & 0 & 0 & - & 2.7 & - & 42.5 & 32.9 & 66.7 & 5.3 \\
\hline $26[0-20 \mathrm{~cm}]$ & 0 & 41 & 0 & 0 & 0 & 0 & 0 & 41 & 9 & 50 & 37 & 17 & - & 5 \\
\hline
\end{tabular}


Table 1 (continued)

\begin{tabular}{|c|c|c|c|c|c|c|c|c|c|c|c|c|c|c|}
\hline CK [depth-S] & $\begin{array}{l}\text { Abies } \\
\text { alba t. }\end{array}$ & $\begin{array}{l}\text { Fagus } \\
\text { sylvatica }\end{array}$ & Fraxinus & M. Ericaceae & $\begin{array}{l}\text { Taxus } \\
\text { baccata }\end{array}$ & U. Angio. & U. Gymno. & Total & bark & Total & w/hyphae & w/vitrifi. & $\begin{array}{l}\text { Gymno. } \\
\text { w/CW }\end{array}$ & $\mathrm{w} / \mathrm{RC}$ \\
\hline$(\%-\mathrm{H})$ & 0 & 100 & 0 & 0 & 0 & 0 & 0 & - & 18 & - & 90.2 & 41.5 & - & 10 \\
\hline $27[0-20 \mathrm{~cm}]$ & 5 & 40 & 0 & 0 & 0 & 0 & 0 & 45 & 5 & 50 & 30 & 12 & 0 & 0 \\
\hline$(\%-\mathrm{H})$ & 11.1 & 88.9 & 0 & 0 & 0 & 0 & 0 & - & 10 & - & 66.7 & 26.7 & 0 & 0 \\
\hline $28[0-20 \mathrm{~cm}]$ & 1 & 49 & 0 & 0 & 0 & 0 & 0 & 50 & 0 & 50 & 28 & 24 & 0 & 9 \\
\hline$(\%-\mathrm{H})$ & 2 & 98 & 0 & 0 & 0 & 0 & 0 & _ & 0 & - & 56 & 48 & 0 & 18 \\
\hline $29[20-40 \mathrm{~cm}]$ & 0 & 43 & 0 & 0 & 0 & 0 & 0 & 43 & 7 & 50 & 11 & 21 & - & 2 \\
\hline$(\%-\mathrm{H})$ & 0 & 100 & 0 & 0 & 0 & 0 & 0 & - & 14 & _ & 25.6 & 48.8 & - & 4 \\
\hline $30[0-20 \mathrm{~cm}]$ & 0 & 49 & 0 & 0 & 0 & 0 & 0 & 49 & 1 & 50 & 31 & 31 & - & - \\
\hline$(\%-\mathrm{H})$ & 0 & 100 & 0 & 0 & 0 & 0 & 0 & - & 2 & - & 63.3 & 63.3 & - & - \\
\hline $30[20-40 \mathrm{~cm}]$ & 1 & 44 & 0 & 0 & 0 & 0 & 0 & 45 & 0 & 45 & 15 & 19 & - & _- \\
\hline$(\%-\mathrm{H})$ & 2.2 & 97.8 & 0 & 0 & 0 & 0 & 0 & - & 0 & - & 33.3 & 42.2 & - & - \\
\hline $\mathrm{T}-30$ & 1 & 93 & 0 & 0 & 0 & 0 & 0 & 94 & 1 & 95 & 46 & 50 & 0 & 3 \\
\hline$(\%-\mathrm{H})$ & 1.1 & 98.9 & 0 & 0 & 0 & 0 & 0 & - & 1.1 & - & 48.9 & 53.2 & 0 & 3.2 \\
\hline $31[0-20 \mathrm{~cm}]$ & 9 & 35 & 0 & 0 & 0 & 0 & 0 & 44 & 6 & 50 & 41 & 24 & 3 & 3 \\
\hline$(\%-\mathrm{H})$ & 20.5 & 79.5 & 0 & 0 & 0 & 0 & 0 & - & 12 & - & 93.2 & 54.5 & 33.3 & 6 \\
\hline Total & 217 & 2041 & 1 & 2 & 1 & 1 & 4 & 2267 & 175 & 2442 & 1379 & 861 & 40 & 118 \\
\hline$(\%-H)$ & 9.57 & 90.03 & 0.04 & 0.09 & 0.04 & 0.04 & 0.18 & - & 7.17 & - & 60.83 & 37.98 & 18.02 & 4.8 \\
\hline
\end{tabular}

Table 2

AMS dates on charcoals from The Argentières ancient dump, charcoal kilns (CK) and pedanthracological pit (Lacore 1).

\begin{tabular}{|c|c|c|c|c|c|c|c|}
\hline Sector & Sample & Site & Lab. no. & Material & Taxa & Age $14 \mathrm{C} \mathrm{BP}$ & Date cal BC-AD (95.4\% prob.) \\
\hline Pic des Argentières & 1 & Dump SU4 & Poz-68041 & Charcoal & Abies alba $t$. & $2025 \pm 30$ & $151 \mathrm{cal} B C-55 \mathrm{cal}$ AD \\
\hline Pic des Argentières & 17 & Dump SU3 & Poz-77848 & Charcoal & Abies alba t. + bark & $2200 \pm 30$ & 366-192 cal BC \\
\hline Étang de Labant & 8 & CK 20 & Poz-68048 & Charcoal & Conifer & $370 \pm 30$ & $1447-1634 \mathrm{cal}$ AD \\
\hline Étang de Labant & 7 & CK 18(2) & Poz-68047 & Charcoal & Bark & $355 \pm 30$ & $1453-1635 \mathrm{cal} \mathrm{AD}$ \\
\hline Lacore & 3 & CK 4 & Poz-68043 & Charcoal & Fagus sylvatica & $340 \pm 30$ & $1470-1640 \mathrm{cal} \mathrm{AD}$ \\
\hline Lacore & 16 & CK 10 & Poz-73026 & Charcoal & Fagus sylvatica & $265 \pm 30$ & $1516-1950 \mathrm{cal} \mathrm{AD}$ \\
\hline Pic des Argentières & 2 & CK 1 & Poz-68042 & Charcoal & Fagus sylvatica & $215 \pm 30$ & $1644-1931 \mathrm{cal} \mathrm{AD}$ \\
\hline Coumebière & 9 & CK 24 & Poz-68050 & Charcoal & Fagus sylvatica & $195 \pm 30$ & $1648-1925 \mathrm{cal} \mathrm{AD}$ \\
\hline Lacore & 4 & CK 7 & Poz-68044 & Charcoal & Fagus sylvatica & $170 \pm 30$ & $1659-1916 \mathrm{cal} \mathrm{AD}$ \\
\hline Lacore & 14 & CK 22 & UGAMS \# 19688 & Charcoal & Ericaceae + F. sylvatica & $170 \pm 20$ & $1665-1919 \mathrm{cal} \mathrm{AD}$ \\
\hline Bois des Plagnolles & 10 & CK 26 & Poz-68051 & Charcoal & Fagus sylvatica & $135 \pm 30$ & $1671-1943 \mathrm{cal} \mathrm{AD}$ \\
\hline Étang de Labant & 5 & CK $17(1)$ & Poz-68045 & Charcoal & Fagus sylvatica & $115 \pm 30$ & $1680-1939$ cal AD \\
\hline Étang de Labant & 6 & CK 17(2) & Poz-68046 & Charcoal & Fagus sylvatica & $85 \pm 30$ & $1688-1927 \mathrm{cal} \mathrm{AD}$ \\
\hline Lacore & 15 & CK 13 & UGAMS \# 19689 & Charcoal & Fagus sylvatica & $80 \pm 20$ & 1694-1919 cal AD \\
\hline Lacore & 1 & Pit 2 LI & Poz-81264 & Charcoal & Abies alba t. & $760 \pm 30$ & $1219-1284 \mathrm{cal} \mathrm{AD}$ \\
\hline Lacore & 2 & Pit 2 LI & Poz-82892 & Charcoal & Fagus sylvatica & $120 \pm 30$ & $1679-1940 \mathrm{cal} \mathrm{AD}$ \\
\hline Lacore & 3 & Pit 1 LII & Poz-82893 & Charcoal & Fagus sylvatica & $100 \pm 30$ & $1682-1935 \mathrm{cal} \mathrm{AD}$ \\
\hline Lacore & 4 & Pit 1 LV & Poz-82894 & Charcoal & Angiospermae & $9950 \pm 50$ & $9664-9292 \mathrm{cal} \mathrm{BC}$ \\
\hline
\end{tabular}

Table 3

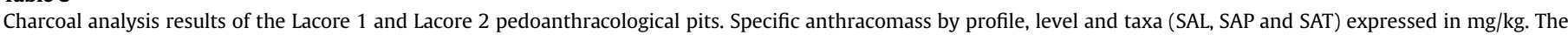
relative values of charcoals affected by vitrification and fungi affectation are expressed in percentage for Abies alba type and Fagus sylvestris.

\begin{tabular}{|c|c|c|c|c|c|c|c|c|c|c|}
\hline $\begin{array}{l}\text { Sampling } \\
\text { point }\end{array}$ & Level & Abies alba t. & Fagus sylvatica & Vaccinium & $\begin{array}{l}\text { Taxus } \\
\text { baccata }\end{array}$ & Angiospermae & Gymnospermae & Undeterminable & SAL & SAP \\
\hline Lacore 1 & $\begin{array}{l}\text { I } \\
\text { II } \\
\text { III } \\
\text { IV } \\
\text { V }\end{array}$ & $\begin{array}{l}2,43(\mathrm{v}=40 \%, \mathrm{~h}=0 \%) \\
3,31(\mathrm{v}=18,2 \%, \mathrm{~h}=2.6 \%)\end{array}$ & $\begin{array}{l}3,41(\mathrm{v}=25 \%, \mathrm{~h}=12.5 \%) \\
13,92(\mathrm{v}=23,1 \%, \mathrm{~h}=12.5 \%)\end{array}$ & $\begin{array}{l}1,95 \\
0,33\end{array}$ & 0,97 & $\begin{array}{l}3,65 \\
1,33 \\
35,37\end{array}$ & $\begin{array}{l}1,22 \\
0,66 \\
1,97\end{array}$ & $\begin{array}{l}1,71 \\
0,66 \\
1,65 \\
7,91\end{array}$ & $\begin{array}{l}44,6 \\
25,86 \\
0 \\
0 \\
45,45\end{array}$ & 18.7 \\
\hline Lacore 2 & $\begin{array}{l}\text { I } \\
\text { II } \\
\text { III } \\
\text { IV }\end{array}$ & $\begin{array}{l}40,77(v=39,3 \%, h=8.9 \%) \\
1,82(v=20 \%, h=0 \%) \\
1,35(v=100 \%, h=0 \%)\end{array}$ & $80,44(v=47,8 \%, h=31.1 \%)$ & & & 3,86 & 1,1 & $\begin{array}{l}4,95 \\
4,95 \\
10,8 \\
1,65\end{array}$ & $\begin{array}{l}207,71 \\
13,37 \\
13,5 \\
6,62\end{array}$ & 65.6 \\
\hline
\end{tabular}

Pic des Argentières, Southern workshops, and Coumebière. The first area shows an underground working place $(15 \times 4 \mathrm{~m})(\mathrm{H})$ that is blocked at the bottom, and seven opencast works with blasting marks: two attacks ( $A$ and $F$ ), three backfilled trenches (B, C, D), one backfilled shaft (E), and another $(G)$ embedded in a large trench and leading to a waste heap related to reactivation works. The trench $\mathrm{C}$ shows firesetting marks on working faces of both its extremities. It is connected (probably with $\mathrm{D}$ and $\mathrm{E}$ ) to a waste heap with irregular contours. The pedoarchaeological section shows that its zone is disturbed and composed entirely of excavated materials from firesetting (Fig. 3). Radiocarbon dates suggest two former mining phases: during La Tène (366-192 cal BC) and around the turn of the Roman period (151 cal BC-55 cal AD) (Table 2). Three galena samples were discovered at the surface of SU4 stratigraphic bore. Their $\mathrm{Pb}$ isotopic signatures are included between 38.570 and 38.557 , 15.688 to 15.687 and 18.380 to 18.364 for ${ }^{208} \mathrm{~Pb} /{ }^{204} \mathrm{~Pb},{ }^{207} \mathrm{~Pb} /{ }^{204} \mathrm{~Pb}$ 
Table 4

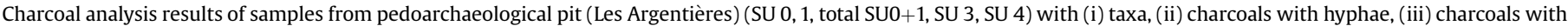
vitrifications, conifers with compression wood absolute (n) and relative frequencies (\%) and Pareto index value.

\begin{tabular}{|c|c|c|c|c|c|c|c|c|c|c|c|c|}
\hline \multirow{3}{*}{$\begin{array}{l}\text { Calibrated dates } \\
\text { (calBC/calAD) } \\
\text { SU - Ancient Dump of } \\
\text { Argentières } \\
\text { Taxa }\end{array}$} & \multicolumn{2}{|c|}{-} & \multicolumn{2}{|c|}{-} & \multicolumn{2}{|c|}{-} & \multicolumn{2}{|c|}{ 366-192 cal BC } & \multicolumn{2}{|c|}{$151 \mathrm{cal} \mathrm{BC}-55 \mathrm{cal} \mathrm{AD}$} & \multicolumn{2}{|c|}{-} \\
\hline & \multicolumn{2}{|c|}{ SU 0} & \multicolumn{2}{|c|}{ SU 1} & \multicolumn{2}{|c|}{ Total SU0-1 } & \multicolumn{2}{|c|}{ SU 3} & \multicolumn{2}{|c|}{ SU 4} & \multicolumn{2}{|c|}{ Total } \\
\hline & $\mathrm{n}$ & $\%$ & $\mathrm{n}$ & $\%$ & $\mathrm{n}$ & $\%$ & $\mathrm{n}$ & $\%$ & $\mathrm{n}$ & $\%$ & $\mathrm{n}$ & $\%$ \\
\hline Abies alba type & 53 & 54.1 & 45 & 45 & 98 & 49.5 & 94 & 47.5 & 55 & 61.8 & 247 & 50.9 \\
\hline Alnus-Betula & 0 & 0 & 0 & 0 & 0 & 0 & 2 & 1 & 0 & 0 & 2 & 0.4 \\
\hline Betula & 1 & 1 & 0 & 0 & 1 & 0.5 & 3 & 1.5 & 0 & 0 & 4 & 0.8 \\
\hline Corylus avellana & 0 & 0 & 0 & 0 & 0 & 0 & 1 & 0.5 & 0 & 0 & 1 & 0.2 \\
\hline Fagus sylvatica & 35 & 35.7 & 44 & 44 & 79 & 39.9 & 83 & 41.9 & 31 & 34.8 & 193 & 39.8 \\
\hline Ilex aquifolium & 1 & 1 & 0 & 0 & 1 & 0.5 & 0 & 0 & 0 & 0 & 1 & 0.2 \\
\hline Leguminosae & 1 & 1 & 0 & 0 & 1 & 0.5 & 0 & 0 & 0 & 0 & 1 & 0.2 \\
\hline Mountain Ericaceae & 0 & 0 & 0 & 0 & 0 & 0 & 1 & 0.5 & 0 & 0 & 1 & 0.2 \\
\hline Pinus uncinata type & 4 & 4.1 & 2 & 2 & 6 & 3 & 3 & 1.5 & 0 & 0 & 9 & 1.9 \\
\hline $\begin{array}{l}\text { Rosaceae, Maloideae } \\
\text { cf. S. aucuparia }\end{array}$ & 1 & 1 & 1 & 1 & 2 & 1 & 1 & 0.5 & 0 & 0 & 3 & 0.6 \\
\hline Taxus baccata & 2 & 2 & 7 & 7 & 9 & 4.5 & 10 & 5.1 & 2 & 2.2 & 21 & 4.3 \\
\hline Und. Gymnospermae & 0 & 0 & 1 & 1 & 1 & 0.5 & 0 & 0 & 1 & 1.1 & 2 & 0.4 \\
\hline Total & 98 & 1 & 100 & 1 & 198 & 1 & 198 & 1 & 89 & 1 & 485 & 1 \\
\hline Indeterminable & 2 & 2 & 0 & 0 & 2 & 1 & 2 & 1 & 11 & 11 & 15 & 3 \\
\hline Total charcoals analysed & 100 & 1 & 100 & 1 & 200 & 1 & 200 & 1 & 100 & 1 & 500 & 1 \\
\hline Charcoals w/hyphae & 31 & 31 & 33 & 33 & 64 & 32 & 61 & 30.5 & 35 & 35 & 160 & 32 \\
\hline Charcoals w/vitrifications & 27 & 27 & 27 & 27 & 54 & 27 & 48 & 24 & 54 & 54 & 210 & 42 \\
\hline $\begin{array}{l}\text { Conifers w/compression } \\
\text { wood }\end{array}$ & 11 & 18.6 & 11 & 20 & 22 & 19.5 & 18 & 16.8 & 12 & 20.7 & 52 & 18.6 \\
\hline Pareto index value & \multicolumn{2}{|c|}{$20 / 80$} & \multicolumn{2}{|c|}{$30 / 70$} & \multicolumn{2}{|c|}{$20 / 80$} & \multicolumn{2}{|c|}{$21 / 79$} & \multicolumn{2}{|c|}{$30 / 70$} & \multicolumn{2}{|c|}{$16 / 84$} \\
\hline
\end{tabular}

and ${ }^{206} \mathrm{~Pb} /{ }^{204} \mathrm{~Pb}$ ratios respectively (Fig. 5). The Southern workshops area includes: an 18th c. collapsed crosscut (TB Sud) connected to a large waste heap and ruins of three buildings (B1, B2, B3), as well as a mineral ore processing area with two waste heap zones. The Coumebière area comprises a research trench and a small dump that probably date from the end of the 19th c.

Lacore sector. Mining remains are scattered across the northern slope on a surface of $150 \times 150 \mathrm{~m}$. Three mining areas were distinguished: the Versant, Pic and Gorge areas (Fig. 2). The Versant area saw the bulk of the work (Mine of Lacore A) that has exploited the ore vein principally during the 18 th $\mathrm{c}$. It includes a long adit (crosscut A) that opens into a large shaft and leads to a drift following the vein. Inside crosscut A, six galena have been sampled during the fieldwork. Their $\mathrm{Pb}$ isotopic signatures are included between 38.725 and $38.707,15.695$ to 15.694 and 18.502 to 18.490 for ${ }^{208} \mathrm{~Pb} /{ }^{204} \mathrm{~Pb},{ }^{207} \mathrm{~Pb} /{ }^{204} \mathrm{~Pb}$ and ${ }^{206} \mathrm{~Pb} /{ }^{204} \mathrm{~Pb}$ ratios respectively (Fig. 5). The drift overhangs an underground working place (not measured) and leads to a chaotic karst zone in the West. It crosses a sterile area which leads to a collapsed shaft in the east (D). In the last few metres, a decline provides access to inferior important works (not measured) dating from the 19th c. The drift B dug during the same period gave access to this part of the mine. It was preceded by an unfinished crosscut B mentioned in manuscript of the 18th c. The Pic area is comprised of small open cast exploration works (scraping, cap drifts and small dump) with drill traces. The Gorge area includes a modern drift $G$ cross driving the bottom of two narrow vertical tunnels that were sunk before the use of explosive powder.

\subsubsection{Charcoal kilns}

Thirty-one CK ranged at altitudes from 1.400 to $1.650 \mathrm{~m}$ have been discovered (Py et al., 2013a) (Fig. 2). Their density is relatively low (at least 0.6 per ha) with the understanding that the area of wood supply for a charcoal kiln in Mediterranean French forest is around 0.785 ha (Fabre, 1992). Most of them are located under forest cover. Some of them are located on the margin of Coumebière, but none were found on the plateau. One in five is beside or on a path that is still visible. Their contours are rarely clearly evident or preserved. Their sizes that were valued by pedological coring vary quite substantially: $2.5-3 \times 3 \mathrm{~m}$ for the smallest ones and $5 \times 6 \mathrm{~m}$ to $7 \times 8 \mathrm{~m}$ for the largest ones. The smaller ones occupy confined sloping ledges on heavy slopes. The charcoal layer is generally thin $(10-15$ to $20-40 \mathrm{~cm})$. It is usually located directly under the humus layer. ${ }^{14} \mathrm{C}$ dates of the oldest ones (CK 20, 18, 4, 10) are ranged between 1447 and 1640 cal AD (85.9-95.4\% prob.) (Table 2). The others present ${ }^{14} \mathrm{C}$ ages distributed between 1644 and 1950 cal AD (95.4\% prob.), i.e. on the modern radiocarbon plateau (Table 2 ).

\subsection{Charcoal analysis results}

\subsubsection{Charcoal kilns (CK)}

In total, there are 7 taxa identified: Abies alba type (silver fir on the basis of ecology in our study area), Fagus sylvatica, Fraxinus, mountain Ericaceae (Rhododendron ferrugineum and/or Vaccinium), Taxus baccata, undeterminable Angiospermae and Gymnospermae (Table 1 and Fig. 6). The corpus is largely dominated by beech (relative frequencies between 62 and 100\%), alone (in CK 9, 12, 13, $24,26,29$ ) or in association with fir (from 1.1 to $36 \%$ ). Ash is only present in CK 10 (2\%), yew in CK 1 (0.6\%), mountain Ericaceae in CK $3(1.2 \%)$ and $22(2 \%)$ and their relative frequencies are very low indeed anecdotal. The chronological classification of dated CK shows that the oldest ones (CK 18, 20, 10 et 4) have significantly higher fir frequencies than the most recent ones (Fig. 6). For centuries, the evolution of main taxa (fir and beech) proportions seems linear: the fir declines in favour of beech. The HCPC distributes the $31 \mathrm{CK}$ into three clusters A, B, C (Fig. 7): (i) "A" gathers the CK with higher fir proportions and includes the oldest ones; (ii) " $B$ " and " $C$ " regroup the $\mathrm{CK}$ with ${ }^{14} \mathrm{C}$ dates on the modern radiocarbon plateau but they are divided into two different groups; B with intermediate 


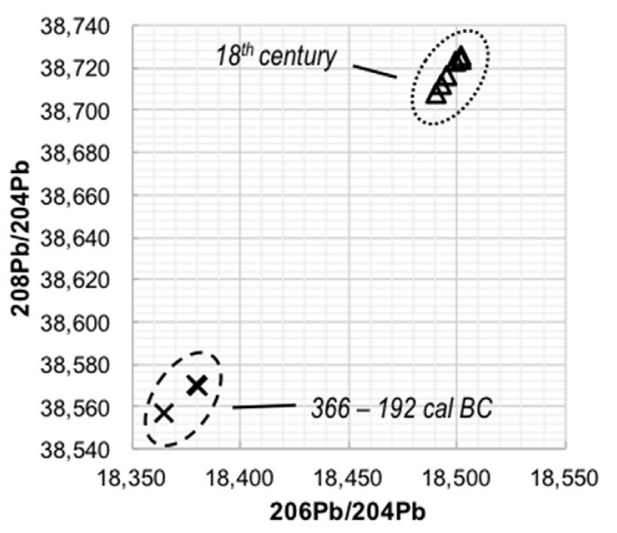

XOres from "Les Argentières" mine $\Delta$ Ores from "Lacore" mine

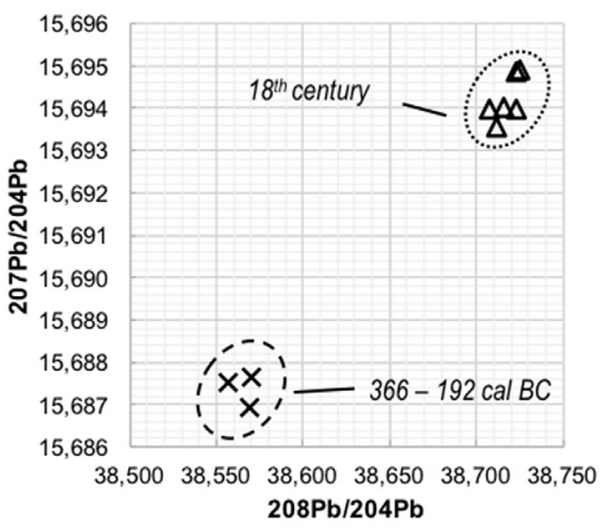

XOres from "Les Argentières"

$\Delta$ Ores from "Lacore" mine

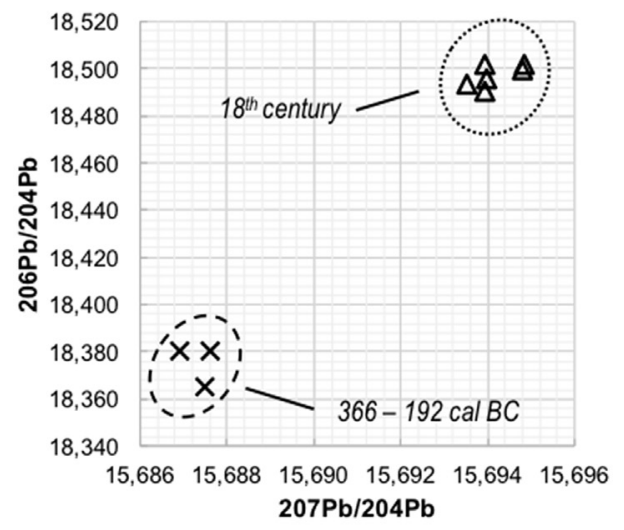

XOres from "Les Argentières" mine

$\Delta$ Ores from "Lacore" mine

Fig. 5. $\mathrm{Pb} / \mathrm{Pb}$ diagrams showing the lead isotopic signatures on galena in Les Argentières and Lacore mines. Les Argentières have been exploited during Iron Age period and Lacore during the 18th century. The error bars (measuring the total external uncertainties per sample) being smaller than the symbol, they have not been reported here.

fir proportions and $\mathrm{C}$ with lower fir proportions. The pairwise comparisons using Wilcoxon rank sum test per taxon (fir and beech) shows a significant difference between the three clusters with $\mathrm{p} \leq 0.001$ (Table 5).
Single bark fragments (undeterminable) were identified in most charcoal kilns (except CK 10, 22, 28) and they constitute a part of the corpus (1.1-18\%). 3.4\% of analysed charcoals $(n=2442)$ have cortical and cambial cells preserved at the last ring (the bark conservation is considered as random). These are essentially beech charcoals (96.2\%). The area of growth stop was identified for all of them $(n=78) ; 78.2 \%$ present a growth stop in the late wood $(13.3 \%$ in the end of the late wood) and $22.8 \%$ in the early wood $(18.7 \%$ in the end of the early wood) (Table 6). For these results to be extrapolated to the entire corpus we must accept a $12 \%$ margin of error that seems to us to be too high. Only twenty charcoals have bark and pith preserved. Their minimal initial diameters are between 0.2 and $3 \mathrm{~cm}$ (average $=0.7 \mathrm{~cm}$ ).

All samples include charcoals with hyphae contamination. The contaminated charcoal frequencies are variable, but they are frequently greater than $50 \%$ (average $=60.8 \%$ ) (Table 1 ). In most cases, the colonisation of cellular structures by hyphae is low to moderate. Charcoals altered by fungi are, a priori, scarce, but note that we do not have baseline for beech wood from various levels of fungi colonisation. All samples also include charcoals with vitrifications (local to general) for which frequencies are comprised between 15 and 60\% (average $=37.9 \%$ ) (Table 1 ). $4.8 \%$ of charcoals present radial cracks (rarely more than one on the transverse plane) (Table 1). Lastly, frequencies of conifer charcoal with compression wood are comprised between 0 and 66\% (average $=18.02 \%$ ) (Table 1). These latter results have to be interpreted with care because the number of conifers is generally very small.

\subsubsection{Pedoarchaeological pit (Les Argentières)}

A total of 12 taxa were identified: Abies alba type, Alnus-Betula, Betula, Corylus avellana, Fagus sylvatica, Ilex aquifolium, Leguminosae, mountain Ericaceae, Pinus uncinata type (P. uncinata and/or P. sylvestris), Rosaceae, Maloideae cf. Sorbus aucuparia, Taxus baccata, undeterminable Gymnospermae (Fig. 3 and Table 4). The corpus is largely dominated by two arborescent taxa: the fir with a total of $50.9 \%$ and the beech with $39.8 \%$. The European yew is in third position (4.3\%) and pine in fourth (1.9\%). The other taxa have relative frequencies below $1 \%$. The Pareto index value calculated for the whole corpus is relatively high (16/94) indicating a small overrepresentation of the more frequent taxa. By contrast, the index obtained for both superficial units cumulated (SU 0-1) and the SU 3, are close or equal to 20/80. Slightly more than one third of charcoals have hyphae present in their cellular structure but their occurrence is low and moderate. Slightly less than half of charcoals present vitrifications, which is generally partial and localised. 18.6\% of conifer charcoals have compression wood (Table 4).

\subsubsection{Pedoanthracological pits}

Soil charcoal analysis shows that anthracomass (quantity of charcoal per kilogram of dried soil) is present at both sampling points, but with some noticeable differences (Fig. 4). In Lacore 1, specific anthracomasses are low and aggregated at both superficial sampling levels ( 25.86 and $44.60 \mathrm{mg} / \mathrm{kg}$ ). The beech charcoal from the level II dates back to the $17^{\text {th }}-20$ th c. cal AD (Table 2, Fig. 4). There is no anthracomass in both intermediary levels and the inferior one presents the highest mass with $45.45 \mathrm{mg} / \mathrm{kg}$. The Angiospermae fragment from this level (V) dates back to 9664 9292 cal BC (Table 2, Fig. 4). This distribution of anthracomasses assumes a slight risk of charcoal transfers between the surface and depth, and vice versa. In Lacore 2, anthracomass is much higher and is concentrated into the superficial sampling level $(207.71 \mathrm{mg} / \mathrm{kg})$ while it remains very low in the other levels (13.37, 13.50 and $6.62 \mathrm{mg} / \mathrm{kg}$ ) (Fig. 4, Table 3). Moreover, its general anthracomass $(65.6 \mathrm{mg} / \mathrm{kg}$ ) is more than three times higher than that of Lacore 1 . The fir fragment from this level dates back to the 13th c. cal AD and 


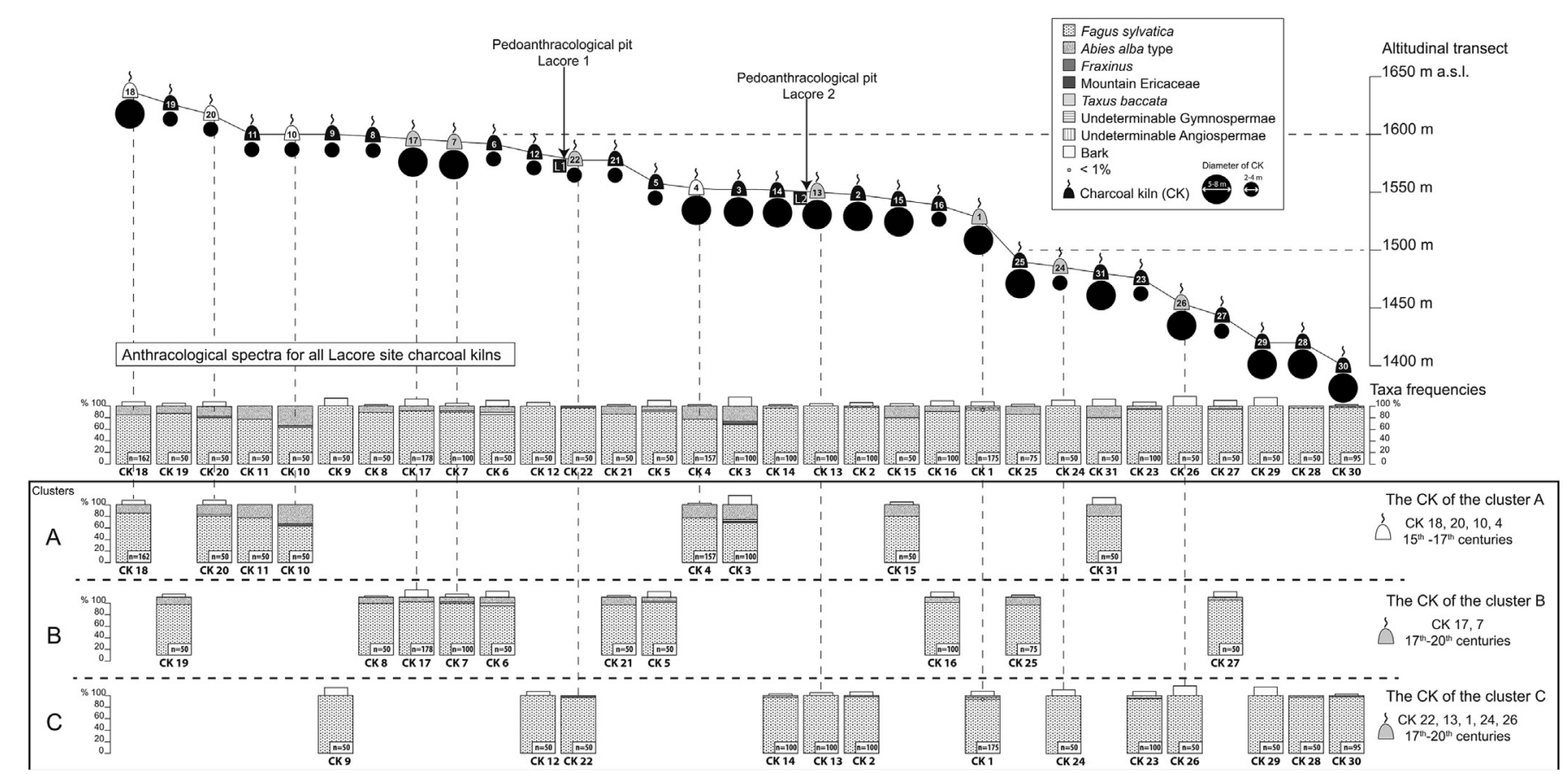

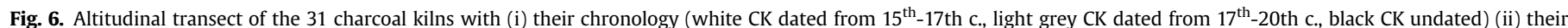

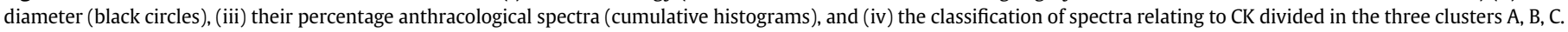

(1) Hierarchical Clustering

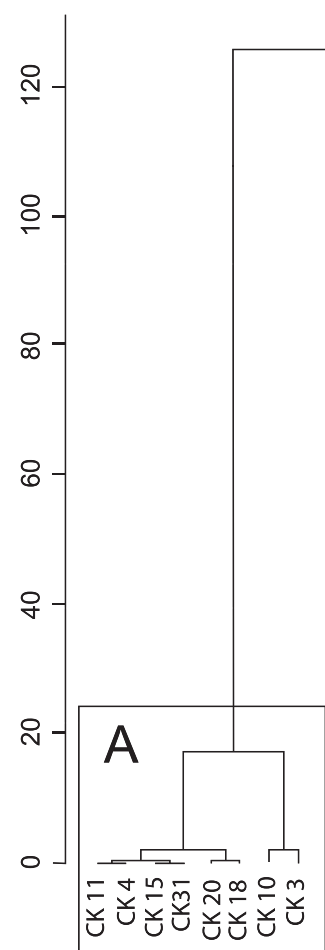

\section{(2) Box-Plot dispersions}

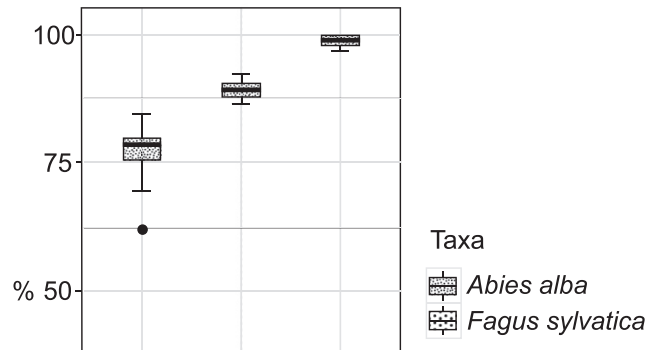

กิ

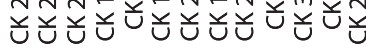

Fig. 7. (1) Hierarchical clustering with the distribution of the $31 \mathrm{CK}$ in three clusters A, B, C and (2) Box-Plot dispersions of Abies alba and Fagus sylvatica proportions in the three clusters.

the beech fragment from the $17^{\text {th }}-20$ th c. cal AD (Table 2, Fig. 4). In total, 6 taxa have been identified: Abies alba type, Fagus sylvatica, Taxus baccata, Vaccinium (V. myrtillus and/or uliginosum), undeterminable Angiospermae and Gymnospermae (Table 3, Fig. 4). Most charcoals extracted and identified are from the $0.8 \mathrm{~mm}$ mesh (Fig. 8). The data expressed in relative frequencies show the 
Table 5

Pairwise comparisons using Wilcoxon rank sum test based on proportions of the main taxa fir and beech per cluster A, B and $C\left({ }^{*}=p\right.$-value $\left.p \leq 0.001\right)$.

\begin{tabular}{lllll}
\hline Clusters & \multicolumn{2}{c}{ A } & \multicolumn{2}{c}{ B } \\
\hline Taxa & F. sylvatica & A. alba & F. sylvatica & A. alba \\
B & $4.6 \mathrm{e}-05^{*}$ & $4.5 \mathrm{e}-04^{*}$ & - & - \\
C & $1.6 \mathrm{e}-04^{*}$ & $1.5 \mathrm{e}-04^{*}$ & $5.4 \mathrm{e}-05^{*}$ & $5.0 \mathrm{e}-05^{*}$ \\
\hline
\end{tabular}

codominance of Abies alba and Fagus sylvatica in all superficial sampling levels from both pits, without taking into account the undeterminable. In Lacore 1, tiny twigs from deciduous species largely dominate in the lower level. Bilberry and yew have been found only in the two superficial levels. In Lacore 1, the beech slightly dominates and it is outclassed by fir in Lacore 2 . This observation is somewhat different for data from each mesh expressed as absolute frequencies: fir dominates in the $0.8 \mathrm{~mm}$ mesh from the Lacore 1 level II but beech dominates in the $2 \mathrm{~mm}$ mesh from the Lacore 2, level I (Fig. 8). The Specific taxonomic Anthracomass per Taxon (SAT) shows the beech dominance in all superficial levels (Fig. 4). These data could suggest a differential fragmentation of both main taxa. Beech, which has fewer fragments from 0.8 to $2 \mathrm{~mm}$ meshes (Lacore 2), has higher masses (Fig. 8). Both taxa are impacted by vitrification phenomenon with no significant difference between proportions of fir and beech fragments vitrified (Table 3). In the same way, hyphae are present in both taxa, but the beech is more affected by wood rotting-fungi (Table 3).

\section{Discussion}

\subsection{New insights on mining and charcoal making history in the Aulus Mountain}

Our investigation reveals that mining emerged during the Iron Age (La Tène), a period sparsely documented by archaeological knowledge available in our study area. In view of the thinness of the waste heap, it was probably a modest activity, which used firesetting and focused on mineralised outcrops. However the expansion of this activity cannot be evaluated. These works were clearly subjected to a reactivation around the turn of the Roman era. The "ancient" dump stratigraphy (with oldest deposits onto more recent ones) suggests the clearing of former works before a new extraction phase. These chronological indices correlate well with palaeoenvironmental and archaeological data obtained in the Basque Mountains (Galop et al., 2001; Beyrie et al., 2003; Monna et al., 2004b). The lead isotopic composition of this Iron Age activity is strongly less radiogenic than the more recent one dated from the 18th c. More broadly, the study of this early mining activity will be useful to attribute a relevant signature of heavy metal emissions recorded in peat core deposits in Pyrenees (research in progress). Indeed, usually, at a European scale, the so-called "roman peak" is evoked to explain heavy metal records around the first c.

Table 6

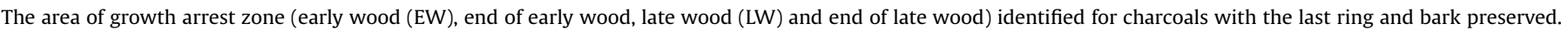

\begin{tabular}{|c|c|c|c|c|c|c|}
\hline TAXA & Early Wood & End of EW & Late Wood & End of LW & Total & $(\%-\mathrm{T})$ \\
\hline Fagus sylvatica & 3 & 14 & 48 & 10 & 75 & 96.2 \\
\hline$(\%-\mathrm{H})$ & 4.0 & 18.7 & 64 & 13.3 & - & - \\
\hline$(\%-V)$ & 100 & 100 & 94.1 & 100 & - & - \\
\hline Abies alba & 0 & 0 & 2 & 0 & 2 & 2.6 \\
\hline Mountain Ericaceae & 0 & 0 & 1 & 0 & 1 & 1.3 \\
\hline Total & 3 & 14 & 51 & 10 & 78 & - \\
\hline$(\%-\mathrm{T})$ & 3.8 & 17.9 & 65.4 & 13 & - & - \\
\hline & \multicolumn{2}{|c|}{$\begin{array}{c}\text { Total Early Wood } \\
\qquad 17\end{array}$} & \multicolumn{2}{|c|}{ Total Late Wood } & & \\
\hline$(\%-\mathrm{T})$ & \multicolumn{2}{|c|}{21.8} & \multicolumn{2}{|c|}{78.2} & & \\
\hline
\end{tabular}

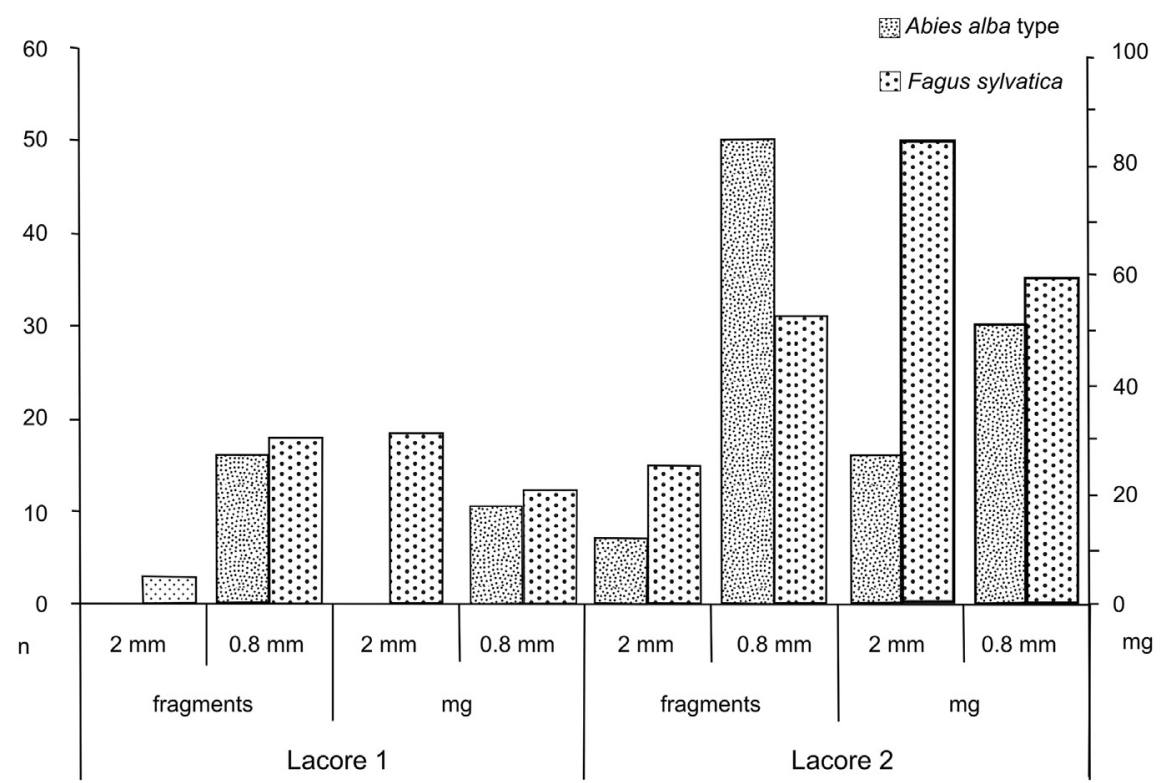

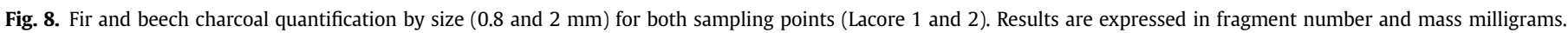


AD in peat deposits (e.g. Le Roux et al., 2004; De Vleeschouwer et al., 2007).

There is locally no concrete evidence of medieval mining, while significant activity has taken place down in the valley in CastelMinier (Verna, 1996; Téreygeol, 2007). The name "Argentières" being typical of Western Europe medieval mines (Bailly-Maître, 2002), one can assume that medieval miners knew these ancient mines but all ore that could be mined using "old technologies" (i.e. firesetting) had been previously exploited. This assumption is based on a constant situation in mining history in Europe as the rich and attainable parts of ore deposits were extracted during the ancient and medieval periods. Mining was reactivated when technological developments ensured the obtention of ore deposits which had previously been difficult to exploit. As elsewhere in the Pyrenees, and more broadly in European mountains, mining activity restarted during the second half of the 17th c., and more importantly from the 18th c. and the 19th c. but, in the Aulus Mountain, for brief periods and very little profits (Braunstein, 1990; Dubois, 1999; Py et al., 2013a). In this way, the lead isotopic composition of the 18th c. mining activity presents a very different lead signature comparing that one of the Iron Age i.e. it is more radiogenic (Fig. 5). These two distinct lead isotopic signatures are due to the geological history of the ores implementation in the Pyrenees (Munoz et al., 2016). The strong differences of lead isotopic compositions between these two mining activities are good indicators to the origins of heavy metals in peat deposits in the Pyrenees. It also allows us to understand if the sources of excess metal are local, regional or distal through comparison of the mean of lead isotopic composition measured in peat deposits.

A first charcoal-making phase took place between the 15th and 17 th c., i.e. at least one century after the signing of the iron-charcoal treaty. The older CK studied by Davasse, 2000 (162-163) in the Vicdessos were from this period and located in the upper section of the valley. The charcoal produced on the Lacore side could supply the Castel-Minier mouline during the $15^{\text {th }}-16$ th $\mathrm{c}$., thus in its last operation phase. That means that it provided itself previously in lowland forest. Furthermore, we cannot exclude that the charcoal production from the Aulus Mountain during the $15^{\text {th }}-17$ th $\mathrm{c}$. was partially or totally devoted to the Vicdessos. One can infer from this data several conclusions concerning to the first-time application of the iron-charcoal treaty: (i) that it was not accepted and applied after its promulgation by the Aulus community known for its independence from the central power (Chevalier, 1956); (ii) that the forests exploited during the 14th c. for the treaty requirements were situated in other valleys of the Couserans (as the Port de Lers high valley) and/or in the lower section of the Aulus valley; (iii) that the charcoal kilns that would be related to the early phase of the treaty application were located at the east of our study area. The extension of the same study to this part of the Aulus Mountain and the lower section of the Aulus valley would respond to this issue in the future. In our study area, the second charcoal-making phase occurred between the $17^{\text {th }}-20$ th c. The clusters B and C (Fig. 6) could characterise two successive phases impossible to situate with certainty on the time scale. The cluster B, with still significant fir proportions, could be the oldest one. In 1762, a text reports that woodlands situated at the western entrance of the Vicdessos were depleted (Davasse, 2000). The iron-charcoal exchange completely ceased in the 1780 s and the Aulus forge stopped in 1820s. Consequently, the $\mathrm{CK}$ of the cluster B could correspond to a period before the second half of the 18th c. and those of the cluster $\mathrm{C}$ to a period after that. Note that in this last phase, the reactivation of mining activity in Les Argentières and Lacore could necessitate charcoal production to supply a forge and test ores. The three buildings situated in the Southern Workshop and dated from the 18th c. probably housed these utilities (Fig. 2).

\subsection{Are soil charcoal assemblages disconnected from charcoal- making?}

The anthracomass values of Lacore 1 and 2 may be considered low, in comparison with those obtained in subalpine and mountain Pyrenean areas (Cunill et al., 2015). In particular, they are relatively close to those acquired from sampling points carried out around the treeline in Southern Pyrenees and Alps (2.000-2.500 m a.s.l.) (Cunill, 2010; Cunill et al., 2012, 2013; Talon, 2010). In these studies, charcoals from superficial levels with high anthracomass generally date from Late Holocene period and they are interpreted as originating from anthropogenic fires. In our study, the fir and beech charcoals from the superficial levels of both sampling points also date from the Late Holocene (13th c. and $17^{\text {th }}-20$ th c.) (Table 2 and Fig. 4). The main taxa proportions (relative values) clearly diverge from anthracological spectra of the neighbouring CK (13 and 21, 22) exclusively dominated by beech and dating from the modern period (Fig. 6). However, the dated beech fragments from Lacore 1 and 2 date back to the second charcoal-making phase (see $\S$ 4.1.). The CK 13 and 22 also date back to this phase (Table 2). Therefore, notwithstanding the low-value of anthracomasses, we cannot exclude beech charcoal transfer from charcoal kilns to superficial soils. However, neighbouring charcoal kilns did not substantially contribute to the significant increase of soil anthracomasses. Moreover, we can see here the complementarity between both anthracological approaches that assume a preferential selection of beech for charcoal-making performed in a forest where fir was maintained during the medieval period and the first part of the modern times (see § 4.3). In both pits' superficial levels, the contribution from shrub and heath formations is limited. The yew could come from undergrowth of a fir, fir-beech or beech closed forest. Thus, it is doubtful that soils charcoals are evidence of pastoral fires carried out in the Lacore side. In the Pyrenees, the practice of pastoral fire aims to burn heath formations to maintain pastures encroached on the forest (Métailié, 2006). In our study area, this practice continues to this day in pastures extended on the other side of the ridge (south-facing). During the field survey, fresh burning marks were found all along the ridge. We can thus reasonably assume that a portion of charcoals found in superficial levels of Lacore 1 and 2 could come from afforestation accidently burned by ill-controlled use of fire practiced on the south-facing slope during the medieval (13th c.) and modern periods (Table 2 and Fig. 4). In this sense, at the regional scale, both periods correspond to high fire frequency mainly controlled by agro-pastoral activities (Rius et al., 2009, 2011, 2012). The action of the wind and the proximity of the Lacore ridge could generate crown fire changed into a surface fire. Fir and beech charcoals infested by hyphae may then come from deadwood having stayed on forest soil. Natural and/or artificial pruning and/or other silvicultural practices were able to produce this deadwood and beech woods seem to be the most affected by them (Table 3 ).

The very humid climate of this area does not favour fire spread even if fire may occur through lightning in forests. This seems to minimise the contribution of natural fires to anthracomass values from superficial levels. Only vitrified charcoals (undeterminable Angiospermae twigs) from the level $\mathrm{V}$ of Lacore 1, dating from the Early Holocene, certainly originate from natural fires. At the regional scale, this period is characterised by increasing drought and summer temperature combined with increasing woodland biomass favouring a higher fire activity (Rius et al., 2014). Locally, charcoal analysis suggests that the vegetation cover was scarcely populated by trees and probably dominated by deciduous shrubs. 


\subsection{Late Holocene woodland cover changes and wood uses}

In local and regional palynogical records, the Iron Age corresponds to an intensification phase of the anthropisation process with increasing of fir forest deforestation (initiated during the Bronze Age), beech expansion and continuance of anthropogenic indicators (Galop and Jalut, 1994; Galop, 1998 and Fig. 9). In the Eastern Pyrenees, previous studies showed that climatic changes, which have occurred since the end of the Bronze Age, have not played a prominent role in opening the landscape or in vegetation changes. Instead, these environmental changes are the result of the combined effect of regular fires, permanent agro-pastoral activities and increasing of human pressure (Galop et al., 2007; Carozza and Galop, 2008; Rius et al., 2012). In this context, our results might suggest a coincidence between the starting point for mining and the conquering of new agro-pastoral terroirs in high mountain areas. During the Second Iron Age (La Tène), the forest exploited for mining (firesetting) is dominated by fir associated with beech. The significant presence of altitudinal pioneer species (Mountain pine and birch) assumes a wood supply in afforestation close to the tree line, probably upstream of the Pic des Argentières and in the Labant glacial cirque. The anthracological spectra of the waste heaps imply the existence of a structured fir-beech forest where fir was locally dominant (Fig. 3). The yew, which has since disappeared (see chapter 2.3, Table 6 and Fig. 6), has broad ecological amplitude and it could have constituted the undergrowth of an Atlantic fir-beech forest with holly, hazel, ash and ericaceous shrubs (Bergmeier et al., 2010). Throughout southwestern Europe, the yew reduction and its gradually disappearance from middle altitudes since ca. 1050 BCE are clearly linked to the double effect of climatic changes and increasing human pressure (Uzquiano et al., 2015). In our study area, charcoal analysis shows that it was weakly present until the modern period and its disappearance seems linked to intensification of agro-sylvo-pastoral practices (Fig. 4).

During the Second Iron Age, it is difficult to characterise the mining impact on forest cover because the magnitude of the works could not be estimated. Together with the extension of agropastoral areas suggested by palynological data, to a certain extent, mining might have contributed to fir and yew decrease and beech progression, as it is a more competitive species. The moment when beech took precedence over fir is difficult to capture. Pollen data place it during the end of the Iron Age and the first centuries of the Roman era (Fig. 9) (Galop, 1998). But locally, charcoal analysis reveals that fir was dominant at least up to the 1st c. AD. Palynological records suggest the maintenance of a fir-beech forest cover until the High Middle Ages (Fig. 9). Simultaneously, the increase of pollen indicators of human activities suggests an expansion of agropastoral activities (Galop, 1998). The pedoanthracological data tend to confirm and specify this hypothesis with south-facing slopes devoted to agro-pastoral activities and north-facing slopes to forestry (Cunill et al., 2015). Locally and around the Couserans, fir pollen curves brutally fall during the Late Middle Ages (Kenla, 1977; Reille, 1993; Galop, 1998 and Fig. 9), which corresponds to the implementation phase of the iron-charcoal treaty and the operation phase of several moulines including Castel-Minier, located in the valley. The charcoal analysis of the former charcoal kilns reveals that fir is still present but it constitutes a modest proportion (about one fifth) of the total wood used. Its frequencies decrease and disappear completely during the modern period (Figs. 6 and 9). In our study area, the significant decrease of pollen fir during the medieval period cannot be directly attributed to charcoal making activity because it occurred later, between the end of the 15th c. and the first half of the 17 th $c$. Here we must probably see the cumulative impact of the higher forest harvesting for local people's needs and lumber production. Manuscripts from the mid-14th c. reported the use of fir and pine in woodmills installed on mountain streams

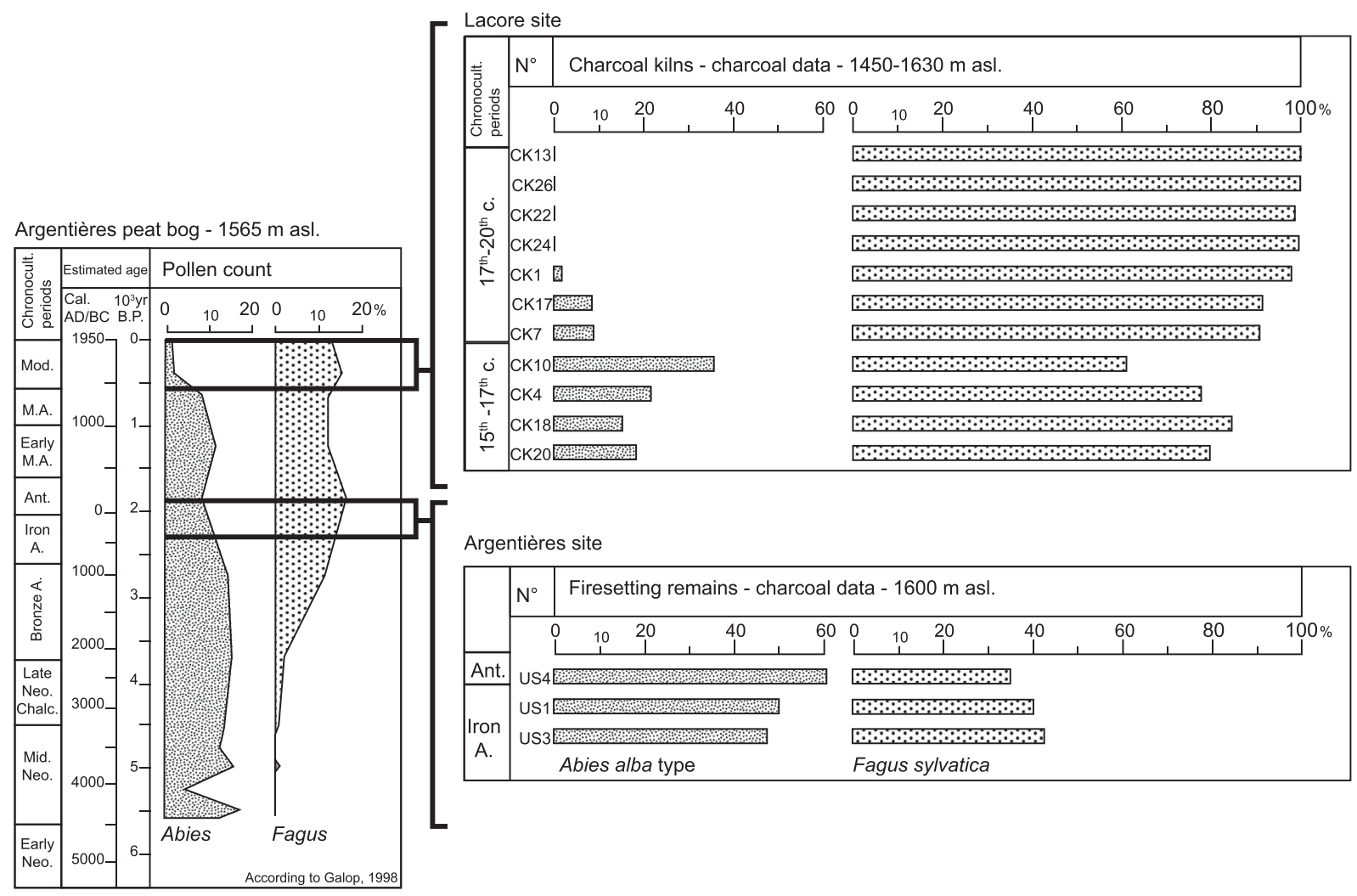

Fig. 9. Parallel display of the palynological data (according to Galop, 1998) against charcoal analysis data. 
such as the brook of Saleix (Verna, 1994, 158). Thus, the full conversion of the fir-beech forest into a single-beech forest occurred during the modern period, probably after the 17 th c., but it was well underway in the late medieval period. The fir disappearance suggests the stop of lumber activity around the 17th c. The studied charcoal kilns are concentrated in the upper part of the ancient mountain forest belt and some of them, particularly the oldest, are located in the transition with the subalpine level. Fir is attested on the entire altitudinal transect during the former phase (Figs. 2 and 6). Its use to make charcoal could cater for a management that aimed to eradicate it in favour of beech coppices more effective for an intensive charcoal production. This management practice would be maintained during the whole the modern period until the 19th c., which might explain that until recently the treeline progression was blocked at around $1.650-1.700 \mathrm{~m}$ a.s.l., as well as the recent regeneration of the fir. Thus, this woodland area located at the western entrance of the Vicdessos was entirely devoted to charcoal making by the beginning of the modern period. In the 19th c., the maintenance of this woodland cover requires a sustainable management of the wood resources set up by the Aulus community. Our results, which must be strengthened by new analyses, assume that the felling activity was mainly focused during autumn and winter, i.e. at this altitude from August or September. The wood was not charred immediately after cutting but most probably in the following autumn thus allowing the contamination of wood by fungi during the short summer season (Py et al., 2013a,b; PySaragaglia et al., 2015). This drying phase of the wood is also suggested by the low frequencies of charcoal with radial cracks. The next estimation of the age and initial diameters of charred wood will allow to better characterise the modalities of this management and in particular the rotation of beech coppices.

\section{Conclusions}

The silver mining remains of Les Argentières and Lacore sites in the Aulus Mountain district have been studied by means of archaeology, history and geochemistry. The archaeological investigation allowed the accurate chronology of the mining works, dating back to the Second Iron Age (the oldest attestation of mining in the Eastern Pyrenees), and the identification of the ores exploited during this former phase and the modern period (18th c.). The coupling of mining archaeology, geology and geochemistry conducted in this project allows us to refine the signature of main mining phases. This demonstrates the relevance of working with a real interdisciplinary approach i.e. the isotopic tracing will help to refine future archaeological, historical and environmental issues on this mining area. It will also be useful to characterise sources of heavy metals recorded in Pyrenean peat deposits that are currently under study. The charcoal analysis of deposits from firesetting allowed the restitution of wood procurement in a structured firbeech forest still dominated by fir and close to the tree line. The cross-study of pollen data from a local peat record; archaeological evidence, and charcoal analysis suggest the coincidence between the emergence of mining activity during the Second Iron Age and the conquering of new high mountain agro-pastoral areas. Together they have contributed to fir decrease and beech progression. The dating and charcoal analysis of the thirty-one charcoal kilns revealed that charcoal-making activity began between the 15th and the 17th c., it probably increased after the 17th c. and continued until the 19th c. It took place within an environment which was already strongly anthropised: devoted to agro-sylvo-pastoral activities and punctually mining. The study of charcoal records from forest soils and charcoal kilns suggests (i) a weak contribution of charcoal making activity to the soil charcoal concentration, (ii) the use of fire to maintain pasture encroached on forest that extended over southern sides, and (iii) the presence of silvicultural activities in the northern ones. In these sloping sides, charcoal makers have gradually eliminated the fir in favour of monospecific beech woodlands, which were maintained and managed until the complete stop of metallurgical activity. Hence, these woodlands located in the Port de Saleix area fuelled the mouline and forges installed in the Upper Garbet Valley and they have also contributed to the ironcharcoal exchange from the turn of the modern period and probably mainly from the 17 th to the end of the 18 th c. i.e. when the exchange treaty was stopped. The Aulus community has well preserved this central resource for the mountain economy. Ongoing dendroanthracological studies will allow more accurate characterisation of the modalities of this management.

\section{Acknowledgements}

The LABEX DRIIHM and the Observatory Human/Environment Vicdessos (OHM Vicdessos) fund this research in the framework of the project called FODYNA (FOrêts passée, DYnamiques et processus de recolonisation à travers l'étude des activités miNières et métAllurgiques historiques du Haut-Vicdessos) coordinated by Vanessa Py-Saragaglia. The observatory device coordinated by Didier Galop is located in the Vicdessos valley. It allows the development of long-term interdisciplinary studies. We thank the CNRS laboratories GET (i.e. Geosciences-Environnement-Toulouse) and GEOLAB (i.e. Géographie Physique et EnvironnementaleLimoges) and the Poznan Radiocarbon Laboratory (Poland) for their contribution. We thank the Regional Archaeological Service of Midi-Pyrénées and the Aulus-les-Bains commune for allowed to the field survey. We are grateful to Clara Siverson for English editing of the manuscript. We thank Chiara Rota and Émilie Dubreucq for their fruitful contribution on the field. The comments of two anonymous reviewers greatly helped to improve the manuscript.

\section{References}

Bailly-Maître, M.-Ch., L’argent, 2002. du minerai au pouvoir. Picard, Paris.

Bal, M-C., Allée, Ph., Liard, M., 2015. The origins of a Nardus stricta grassland through soil charcoal analyses: reconstructing the history of a mountain cultural landscape (Mont Lozère, France) since the Neolithic. Quat. Int. 366, 3-14.

Bergmeier, E., Petermann, J., Schröder, E., 2010. Geobotanical survey of woodpasture habitats in Europe: diversity, threats and conservation. Biodivers. Conserv. 19 (11), 2995-3014.

Beyrie, A., Galop, D., Monna, F., Mougin, V., 2003. La métallurgie du fer au Pays Basque durant l'Antiquité. État des connaissances dans la vallée de Baigorri (Pyrénées-Atlantiques). Aquitania 19, 49-66.

Bonhôte, J., Davasse, B., Dubois, Cl., Izard, V., Métailié, J.-P., 2002. Charcoal kilns and environmental history in the eastern Pyrenees (France). A methodological approach. In: Thiébault, S. (Ed.), Charcoal Analysis. Methodological Approaches, Palaeoecological Results and Wood Uses. Proceedings of the Second International Meeting of Anthracology, Paris, September 2000, BAR International Series, vol. 1063 , pp. $219-228$.

Bonhôte, J., Fruhauf, Ch., 1990. La métallurgie au bois et les espaces forestiers dans les Pyrénées de l'Aude et de l'Ariège. In: Woronoff, D. (Ed.), Forges et forêts, Recherche sur la consommation protoindustrielle de bois. EHESS, Paris, pp. 151-212.

Bonhôte, J., Vernet, J.-L., 1988. La “mémoire des charbonnières". Essai de reconstitution des milieux forestiers dans une vallée marquée par la métallurgie Aston, Haute-Ariège. Rev. For française 40, 197-212.

Braunstein, Ph., 1990. L'industrie minière et métallurgique dans l’Europe médiévale: approche historique et approche archéologique. In: Mannoni, T., Molinari, A. (Eds.), Scienze in Archeologia, Firenze, pp, 143-170.

Breitenlechner, E., Hilber, M., Lutz, J., Kathrein, Y., Unterkircher, A., Oeggl, K., 2010 The impact of mining activities on the environment reflected by pollen, charcoal and geochemical analyses. J. Archaeol. Sci. 37, 1458-1467.

B.R.G.M., 1983. B.R.G.M. "Inventaire minéralogique de la France ${ }^{\circ}{ }^{\circ} 11^{\prime}$, collection R. Pierrot, Ariège, tome 1 Bassin versant du Salat, B.R.G.M. 192 p.

Carozza, L., Galop, D., 2008. Le dynamisme des marges. Peuplement et exploitation des espaces de montagne durant l'Âge du Bronze. In: Guilaine, J. (Ed.), Villes, villages, campagnes de l'Âge du Bronze. Errance, Paris, pp. 226-253.

Chabal, L., 1997. Forêts et sociétés en Languedoc (Néolithique final, Antiquité tardive). L'anthracologie, méthode et paléoécologie. DAF 63, Editions de la Maison 
des Sciences de l'Homme, pp. 33-35. Paris.

Chevalier, M. 1956. La vie humaine dans les Pyrénées ariégeoises. Paris, Génin.

Cunill, R., 2010. Estudi interdisciplinari de l'evolució del límit superior del bosc durant el període holocènic a la zona de Plaus de Boldís-Montarenyo, Pirineu central catal_a. Pedoantracologia, palinologia, carbons sedimentaris i fonts documentals. Universitat Autònoma de Barcelona, Barcelona. PhD dissertation.

Cunill, R., Soriano, J.M., Bal, M.C., Pèlachs, A., Pérez-Obiol, R., 2012. Holocene treeline changes on the south slope of the Pyrenees: a pedoanthracological analysis. Veg. Hist. Archaeobotany 21, 373-384.

Cunill, R., Soriano, J.M., Bal, M.C., Pèlachs, A., Rodriguez, J.M., Pérez-Obiol, R., 2013. Holocene high-altitude vegetation dynamics in the Pyrenees: a pedoanthracology contribution to an interdisciplinary approach. Quat. Int. 289, 60-70.

Cunill, R., Métailié, J.-P., Galop, D., Poublanc, S., Munik, N. de, 2015. Palaeoecological study of Pyrenean lowland fir forests: exploring mid-late Holocene history of Abies alba in Montbrun (Ariège, France). Quat. Int. 366, 37-50.

Davasse, B., 2000. Forêts, charbonniers et paysans dans les Pyrénées de l'est du Moyen Âge à nos jours. Une approche géographique de l'histoire de l'environnement. GEODE, Toulouse.

Davasse, B., Galop, D., 1994. Impact des activités pastorale et métallurgique sur les forêts d'altitude dans les Pyrénées ariégeoises (France), Actes du colloque international Ecologie et Biogéographie Alpines, La Thuile (Italie), 1990, 1-6 septembre, Revue Valdotaine d'Histoire Naturelle, supplément $n^{\circ} 8,151-160$.

De Vleeschouwer, F., Gérard, L., Goormaghtigh, C., Mattielli, N., Le Roux, G., Fagel, N., 2007. Atmospheric lead and heavy metal pollution records from a Belgian peat bog spanning the last two millenia: human impact on a regional to global scale. Sci. Total Environ. 377, 282-295.

Dietrich, Ph.-F. de, 1786. Description des gîtes de minerai, des forges et des salines des Pyrénées, suivi d'observations sur le fer mazé et sur les mines des Sards en Poitou. Didot et Cuchet.

Dillmann, Ph., Téreygeol, F., Verna, C., 2006. Premières analyses métallographiques des produits sidérurgiques trouvés sur le site médiéval de Castel-Minier (Aulusles-Bains, 09). Archéosci. Rev. d'Archéométrie 30, 7-14.

Dubatik, Cl., 1981. Recherches sur les travaux miniers du Haut-Salat, 1ère partie : Les mines d'Aulus-les-Bains. Saint-Girons, Impr. Floquet.

Dubois, Cl., 1999. Les mines de plomb argentifère et zinc d'Aulus-les-Bains (Ariège). Archéologie du Midi Médiéval 17, 187-211.

Dubois, $\mathrm{Cl} .$, 2001. Eléments sur l'origine du fer en Ariège: le site de Lercoul. In: Ministeri de Cultura d'Andorra (Ed.), L'obtencio del ferro pel procediment directe entre els segles IV i XIX, actes del 6 è curs d'arqueologia d'Andorra, 2000, Area de Recerca Historica, Andorra, pp. 161-175.

Dubois, Cl., Métailié, J.-P., 1991. Mines, métallurgie et forêts dans les Pyrénées ariégeoises de l'Antiquité au Moyen Âge, Projet collectif de recherche rapport d'activité 1991.

Dubois, Cl., Métailié, J.-P., 2001. Anthropisation et dynamique forestière dans les Pyrénées ariégeoises à l'époque gallo-romaine. L'exemple de la forêt de Lercoul. In: Les ressources naturelles des Pyrénées. Leur exploitation durant l'Antiquité, pp. 7-19.

Fabre, L., 1992. Le charbonnage historique en région méditerranéenne française : approche expérimentale et anthracologique. Mémoire de DEA, Université Paris I, Paris X, Museum National d'Histoire Naturelle, Institut National d'Agronomie, Université Montpellier II, pp. 1-95.

Fox, J., Bouchet-Valat, M., Andronic, L., Ash, M., Boye, T., Calza, S., Chang, A., Grosjean, P., Heiberger, R., Pour, K.-K., Kerns, G.-J., Lancelot, R., Lesnoff, M., Ligges, U., Messad, S., Maechler, M., Muenchen, R., Murdoch, D., Neuwirth, E. Putler, D., Ripley, B., Ristic, M., Wolf, P., Wright, K., 2016. Rcmdr: R Commander. https://cran.rproject.org/web/packages/Rcmdr/index.html. Accessed 17 June 2016.

Galop, D., 1998. La forêt, l'homme et le troupeau dans les Pyrénées. 6000 ans d'histoire de l'environnement entre Garonne et Méditerranée. GEODE, Toulouse.

Galop, D., Jalut, G., 1994. Differential human impact and vegetation history in two adjacent Pyrenean valleys in the Ariège basin, southern France, from 3000 B.P. to the present. Veg. Hist. Archaeobotany 3, 225-244.

Galop, D., Tual, M., Monna, F., Dominik, J., Beyrie, A., Marembert, F., 2001. Cinq millénaires de métallurgie en montagne basque. Les apports d'une démarche intégrée alliant palynologie et géochimie isotopique du plomb. Sud-ouest européen 11, 3-15.

Galop, D., Carozza, L., Marembert, F., Bal, M.-C., 2007. Activités agropastorales et climat durant l’Âge du Bronze dans les Pyrénées : l'état de la question à la lumière des données environnementales et archéologiques. In: CTHS. Eclipse 2 “Emprises et déprises agricoles, expension et régression des sociétés entre 3500 et 2500 BP), Apr 2004, Besançon, France. CTHS, 21 (documents préhistoriques), pp. 107-119.

Henry, A., Théry-Parisot, I., 2014. From Evenk campfires to prehistoric hearths: charcoal analysis as a tool for identifying the use of rotten wood as fuel. J. Archaeol. Sci. 52, 321-336.

Husson, F., Sosse, J., Le, S., 2016. RcmdrPlugin.FactoMineR: Graphical User Interface for FactoMineR. https://cran.r-project.org/web/packages/RcmdrPlugin. FactoMineR/index.html. Accessed 6 January 2016.

Izard, V., 1993. La typologie des charbonnières : méthode d'étude diachronique du charbonnage pour une contribution à l'histoire des forêts. Actes du colloque Proto-industries et histoire des forêts, 11-12 octobre 1990, Foix, Cahiers de l'Isard, vol. 3, pp. 223-236.

Kenla, J.V., 1977. Analyse pollinique de sédiments postglaciaires de l'étang de l'Hers (alt. 1274 m, Ariège), p. 68. Thèse, Université Paul Sabatier.
Kenla, J.V., Jalut, G., 1979. Déterminisme anthropique du développement su hêtre dans la sapinière du Couserans (Pyrénées ariégoises, France) durant le subatlantique. Geobios 12, 235-738.

Knapp, H., 2012. Environmental history of the Harz Mountains during the last 1000 years - combining pollen and charcoal analyses. Quat. Int. 279-280, 249.

Knapp, H., Robin, V., Kirleis, W., Nelle, O., 2013. Woodland history in the upper Harz Mountains revealed by kiln site, soil sediment and peat charcoal analyses. Quat. Int. 289, 88-100.

Knapp, H., Nelle, O., Kirleis, W., 2015. Charcoal usage in medieval and modern times in the Harz Mountains Area, Central Germany: wood selection and fast overexploitation of the woodlands. Quat. Int. 366, 51-69.

Le Roux, G., Weiss, D., Grattan, J., Givelet, N., Krachler, M., Cheburkin, A., Rausch, N., Kober, B., Shotyk, W., 2004. Identifying the sources and timing of ancient and medieval atmospheric lead pollution in England using a peat profile from Lindow bog, Manchester. J. Environ. Monit. 6, 502-510.

Leroy, S., Cohen, S.X., Verna, C., Gratuze, B., Téreygeol, F., Fluzin, Ph., Bertrand, L. Dillman, Ph., 2012. The medieval iron market in Ariège (France). Multidisciplinary analytical approach and multivariate analyses. J. Archaeol. Sci. 39 (4), 1080-1093.

Ludemann, T., 2010. Past fuel wood exploitation and natural forest vegetation in the Black Forest, the Vosges and neighbouring regions in western Central Europe. Palaeogeogr. Palaeoclimatol. Palaeoecol. 291, 154-165.

Marguerie, D., Hunot, J.-Y., 2007. Charcoal analysis and dendrology: data from archaeological sites in western France. J. Archaeol. Sci. 34, 1417-1433.

Métailié, J.-P., 2006. Mountain landscape, pastoral management and traditional practices in the northern pyrenees (France). In: Agnoletti, M. (Ed.), The Conservation of Cultural Landscapes. CABI Books, pp. 108-123.

Métailié, J.-P., Bonhôte, J., Fruhauf, C., 1988. A thousand years of forest history in the french Pyrenees mountains: the Ariege example. In: Human Influence on Forest Ecosystems Development in Europe, Bologne, ESF FERN-CNR, pp. 159-167.

Monna, F., Petit, C., Guillaumet, J.-P., Jouffroy-Bapicot, I., Blanchot, C., Dominik, J., Losno, R., Richard, H., Lévêque, J., Château, C., 2004a. History and environmental impact of mining activity in Celtic Aeduan territory recorded in a peat-bog (Morvan-France). Environ. Sci. Technol. 38, 665-673.

Monna, F., Galop, D., Carozza, L., Tual, M., Beyrie, A., Marembert, F., Chateau, C., Dominik, J., Grousset, F.E., 2004b. Environmental impact of early Basque mining and smelting recorded in a high minerogenic peat deposit. Sci. Total Environ. 327, 197-214.

Moskal-del Hoyo, M., Wachowiak, M., Blanchette, R.A., 2010. Preservation of fung in archaeological charcoal. J. Archaeol. Sci. 37, 2106-2116.

Munoz, M., Baron, S., Boucher, A., Salvi, S., Beziat, D., 2016. Mesozoic vein-type $\mathrm{Pb}-\mathrm{Zn}$ mineralization in the Pyrenees: lead isotopic and fluid inclusion evidence from the Les Argentières and Lacore deposits. Comptes Rendus Geosci. $348,322-332$

Mussy, J., 1864. Notes sur les gîtes métalliques de l'arrondissement de St Girons. Bull. la Société l'Industrie Minérale t. X, 214-221. Pl. VI.

Paradis-Grenouillet, S., Leleu, J.-Ph., Belingard, Ch., Rouaud, R., Allée, Ph., 2010. Un outil pour la simplification des mesures dendrométriques. AnthracoLoJ. In: Panorama de la dendrochronologie en France, EDYTEM coll., 11, Digne, pp. 197-202.

Paradis-Grenouillet, S., Allée, Ph., Servera Vives, G., Ploquin, A., 2015. Sustainable management of metallurgical forest on Mont Lozère (France) during the Early Middle Ages. Environ. Archaeol. 20 (2), 168-183.

Passaqui, B., 1964. Minéralisations d'Aulus-les-Bains (Ariège). Rapport du BRGM dactylo, DRMM 64 A1, p. 45 janvier 1964.

Pèlachs, A., Nadal, J., Soriano, J.M., Molina, D., Cunill, R., 2009. Changes in Pyrenean woodlands as a result of the intensity of human exploitation: 2,000 years of metallurgy in Vallferrera, northeast Iberian Peninsula. Veg. Hist. Archaeobotany $18,403-416$

Py, V., 2006. Mine charcoal deposits : methods and strategies. The medieval Fournel silver mines in the Hautes-Alpes (France). In: Dufraisse, A. (Ed.), Charcoal Analysis : New Analytical Tools and Methods for Archaeology. Papers from the Table-ronde Held in Basel, British Archaeological Reports International Series S, vol. 1483. Archaeopress, Oxford, pp. 35-46.

Py, V., Ancel, B., Métailié, J.-P., Baron, S., Cunill Artigas, R., 2013a. Prospection inventaire $\mathrm{n}^{\circ} 388$, Commune d'Aulus-les-Bains (Ariège). Rapport SRA MidiPyrénées, Toulouse, septembre 2013.

Py, V., Durand, A., Ancel, B., 2013b. Anthracological analysis of wood fuel used for firesetting in medieval metallic mines of the Faravel district (southern French Alps). J. Archaeol. Sci. 40, 3878-3889.

Py, V., Véron, A., Edouard, J.-L., Beaulieu, J.-L., de, Ancel, B., Segard, M., Durand, A., Leveau, Ph., 2014. Interdisciplinary characterisation and environmental imprints of mining and forestry in the upper Durance valley (France) during the Holocene. Quat. Int. 353, 74-97.

Py-Saragaglia, V., Durand, A., Ancel, B., Édouard, J.-L., Walsh, K., Mocci, 2015. Fl., Les dynamiques de la végétation et des anthroposystèmes d'altitude cernées par l'anthracologie pastorale et minière à l'échelle d'un haut vallon alpestre (Freissinières, France). Archeosc. Rev. d'archéométrie 39, 69-92.

Reille, M., 1993. New pollenanalytical researches at Freychinède, Ariège, Pyrenees, France. Diss. Bot. 196, 377-386.

Reimer, P.J., Bard, E., Bayliss, A., Beck, J.W., Blackwell, P.G., Bronk Ramsey, C., Buck, C.E., Edwards, R.L., Friedrich, M., Grootes, P.M., Guilderson, T.P., Haflidason, H., Hajdas, I., Hatté, C., Heaton, T.J., Hoffmann, D.L., Hogg, A.G. Hughen, K.A., Kaiser, K.F., Kromer, B., Manning, S.W., Niu, M., Reimer, R.W. Richards, D.A., Scott, E.M., Southon, J.R., Turney, C.S.M., van der Plicht, J., 2013. 
IntCal13 and Marine13 radiocarbon age calibration curves, 0-50,000 years cal BP. Radiocarbon 55, 1869-1887.

Rius, D., Vannière, B., Galop, D., 2009. Fire frequency and agro-pastoral activities landscape management in the north-western Pyrenean piedmont (France) since early Neolithic (8000 cal. BP). Holocene 19 (6), 1-13.

Rius, D., Vannière, B., Galop, D., Richard, H., 2011. Holocene fire regime changes from multiple-site sedimentary charcoal analyses in the Lourdes basin (Pyrenees, France). Quat. Sci. Rev. 30, 1696-1709.

Rius, D., Vannière, B., Galop, D., 2012. Holocene history of fire, vegetation and land use from the central Pyrenees (France). Quat. Res. 77 (1), 54-64.

Rius, D., Galop, D., Doyen, E., Millet, L., Vannière, B., 2014. Biomass burning response to high-amplitude climate and vegetation changes in Southwestern France from the Last Glacial to the early Holocene. Veg. Hist. Archaeobotany 23 (6) 729-742.

Roepke, A., Krause, R., 2013. High montane-subalpine soils in the Montafon Valley (Austria, northern Alps) and their link to land-use, fire and settlement history. Quat. Int. 308-309, 178-189.

Schweingruber, F.H., 1978. Mikroskopische holzanatomic : Anatomie microscopique du bois. Birsmensdorf, Institut F.d.ral de Recherches Forestières (Suisse), p. 226. Zürcher AG, Zug.

Schweingruber, F.H., 1990. Anatomie europäischer hölzer: Anatomie of European woods. Haupt, Stuttgart, p. 800.

Servera Vives, G. Miras, Y., Riera, S., Julià, R., Allée, Ph., Orengo, H., ParadisGrenouillet, S., Maria Palet, J., 2014. Tracing the land use history and vegetation dynamics in the Mont Lozère (Massif Central, France) during the last 2000 years: The interdisciplinary study case of Countrasts peat bog. Quat. Int. 353 123-139.

Talon, B., 2010. Reconstruction of Holocene high-altitude vegetation cover in the French soutern Alps: evidence from soil charcoal. Holocene 20 (1), 35-44.

Talon, B., Carcaillet, C., Thinon, M., 1998. Etudes pédoanthracologiques des variations de la limite supérieure des arbres au cours de l'Holocène dans les Alpes françaises. Géogr. Phys. Quaternaire 52 (2), 195-208.

Téreygeol, F., 2007. La poursuite des recherches sur le Castel-Minier : un haut lieu de production d'argent, de plomb et de fer ! La Mémoire du Garbet, n³2, pp. $10-17$.

Téreygeol, F., Arles, A., Foy, E., Florsch, N., Llubes, M., 2010. Dosages par fluorescence
$\mathrm{X}$ portable d'ateliers médiévaux de production des métaux non-ferreux : les exemples de Castel-Minier et d'Agnesserre (Aulus-les-bains, 09). Archéosci. Rev. Archéométrie 34, 243-252.

Théry-Parisot, I., 2001. Économie des combustibles au Paléolithique. CNRS, Paris, p. 195.

Théry-Parisot, I., Henry, A., 2012. Seasoned or green? Radial cracks analysis as a method for identifying the use of green wood as fuel in archaeological charcoal. J. Archaeol. Sci. 39 (2), 381-388.

Tolksdorf, JF, Elburg R, Schröder F, Knapp, $\mathrm{H}$, Herbig C Westphal, T, Schneider, B., Fülling, A., Hemker, C., 2015. Forest exploitation for charcoal production and timber since the $12^{\text {th }}$ century in an intact medieval mining site in the Niederpöbel Valley (Erzgebirge, Eastern Germany). J. Archaeol. Sci.:Reports $4,487-500$.

Touflan, Ph., Talon, B., Walsh, K., 2010. Soil charcoal analysis: a reliable tool for spatially precise studies of past forest dynamics: a case study in the French southern Alps. Holocene 20 (1), 45-52.

Uzquiano, P., Allué, E., Antolín, F., Burjachs, F., Picornel, L., Piqué, R., Zapata, L., 2015. All about yew: on the trail of Taxus baccata in southwest Europe by means of integrated palaeobotanical and archaeobotanical studies. Veg. Hist. Archaeobotany 24, 229-247.

Verna, C., 1994. Le temps des moulines. Le fer et son exploitation du comté de Foix à la vicomté de Béarn (fin XIlème-fin XVème siècles), vol. 2. Thèse, Paris, I.

Verna, C., 1996. Une nouvelle page de l'histoire des mines d'argent européennes : le cas des Pyrénées centrales (XIV ${ }^{\mathrm{e}}-\mathrm{XV}^{\mathrm{e}}$ siècles). Bull. de la Société Ariègeoise des Sci. Lettres Arts 201-232.

Verna, C., 2001. Le temps des Moulines. Fer, technique et société dans les Pyrénées centrales (XIIle - XVIe siècles). Publications de la Sorbonne, Paris.

Verna, C., 2008. Moulin à fer, L'Héritage de Bertrand Gille. In: Durand, A. (Ed.), Jeux d'eau. Moulins, meuniers et machines hydrauliques, XIe-XXe siècle, Etudes offertes à Georges Comet, Aix-en-Provence, pp. 273-286.

Vernet, J.-L., Ogereau, P., Figueiral, I., Machado Yanes, C., Uzquiano, P., 2001. Guide d'identification des charbons de bois préhistoriques et récents, Sud-Ouest de l'Europe: France, Péninsule ibérique et Îles Canaries. CNRS, Paris, p. 395.

Wickham, H., Chang, W., RStudio, 2016. ggplot2: an Implementation of the Grammar of Graphics. https://cran.r-project.org/web/packages/ggplot2/index. html. Accessed 1 March 2016 